\title{
A New Type-2 Soft Set: Type-2 Soft Graphs and Their Applications
}

\author{
Khizar Hayat, ${ }^{1}$ Muhammad Irfan Ali, ${ }^{2}$ Bing-Yuan Cao, ${ }^{1,3}$ and Xiao-Peng Yang \\ ${ }^{1}$ School of Mathematics and Information Sciences, Guangzhou University, Guangzhou 510000, China \\ ${ }^{2}$ Department of Mathematics, Islamabad Model College for Girls, F-6/2, Islamabad, Pakistan \\ ${ }^{3}$ Guangzhou Vocational College of Science and Technology, Guangzhou 510550, China
}

Correspondence should be addressed to Muhammad Irfan Ali; mirfanali13@yahoo.com and Bing-Yuan Cao; caobingy@163.com

Received 7 February 2017; Accepted 15 June 2017; Published 18 October 2017

Academic Editor: Katsuhiro Honda

Copyright (C) 2017 Khizar Hayat et al. This is an open access article distributed under the Creative Commons Attribution License, which permits unrestricted use, distribution, and reproduction in any medium, provided the original work is properly cited.

\begin{abstract}
The correspondence between a vertex and its neighbors has an essential role in the structure of a graph. Type- 2 soft sets are also based on the correspondence of primary parameters and underlying parameters. In this study, we present an application of type-2 soft sets in graph theory. We introduce vertex-neighbors based type- 2 soft sets over $X$ (set of all vertices of a graph) and $\mathscr{E}$ (set of all edges of a graph). Moreover, we introduce some type- 2 soft operations in graphs by presenting several examples to demonstrate these new concepts. Finally, we describe an application of type-2 soft graphs in communication networks and present procedure as an algorithm.
\end{abstract}

\section{Preliminaries and Introduction}

A graph $G=(X, \mathscr{E})$ consists of a nonempty set of objects $X$, called vertices, and a set $\mathscr{E}$ of pair of the element of $X$, called edges. Two vertices $x$ and $y$ are adjacent if $x y \in \mathscr{E}$. A simple graph is an unweighted, undirected graph containing no multiple edges or graph loops. A graph $G^{\prime}=\left(X^{\prime}, \mathscr{E}^{\prime}\right)$ is said to be a subgraph of $G=(X, \mathscr{E})$ if $X^{\prime} \subseteq X$ and $\mathscr{E}^{\prime} \subseteq \mathscr{E}$. The neighborhoods of a vertex $x$ in a graph are the set of all the vertices adjacent to $x$ including $x$ itself. The neighbors of a vertex $x$ in a graph are the set of all the vertices adjacent to $x$ excluding $x$ itself. The eccentricity of the vertex $x$ is the maximum distance from $x$ to any vertex. The radius of a graph is the minimum eccentricity of any vertex $x$

Graph theory is rapidly moving into the mainstream of mathematics, mainly because of its applications in diverse fields which include electrical engineering (communications networks and coding theory), computer science (algorithms and computations), and operations research (scheduling). In many cases, it is important to deal with uncertainties using fuzzy sets and logics. The fuzzy graph is a weighted graph which gives a normalized relational strength over a fuzzy subset of a set [1]. Kauffman [2] gave the fundamental definition of a fuzzy graph on the basis of Zadeh's fuzzy relations [3]. Rosenfeld [1] introduced another elaborated definition of fuzzy graphs. Subsequently, Bhattacharya [4] gave some useful results on fuzzy graphs and Mordeson and Nair [5] investigated some operations on fuzzy graph theory. In recent decades, fuzzy graphs were studied by many researchers. Akram et al. extended the concept of fuzzy graphs to bipolar fuzzy graphs [6], intuitionistic fuzzy hypergraphs [7], intuitionistic fuzzy graphs structures [8], and interval valued fuzzy graph [9]. Samanta et al. [10-12] studied vague graphs, fuzzy planar graphs, completeness and regularity of generalized fuzzy graph, and irregular bipolar fuzzy graphs.

Soft set theory [13], firstly initiated by Molodtsov, is a new mathematical tool for dealing with uncertainties. Indeed, Molodtsov's approach shows the applicability of soft sets to several fields and provides some fundamental results subsequently augmented by works like Maji et al. [14] and Aktaş and Cağman [15], among others. Ali et al. [16] presented some new operations in soft set theory and based on the analysis of several operations on soft sets Sezgin and Atagün [17] studied the theoretical aspect of the soft set theory.

Let $A$ be a set of parameters that can have an arbitrary nature (numbers, functions, sets of words, etc.). Let $X$ be a 
universal set and the power set of $X$ is denoted by $P(X)$. The formal definition of soft is defined as follows.

Definition 1 (see [14]). A pair $(S, A)$ is called a soft set over $X$, where $S$ is a mapping given by $S: A \rightarrow P(X)$.

We refer to Molodtsov's soft sets as type-1 soft sets (briefly T1SS). Note that the set of all T1SS over $X$ will be denoted by $\sigma(X)$. We refer to $[13,16,18]$ for basic notions of soft set theory.

The applications of the soft set are progressing rapidly and many researchers are focusing on real and practical problems. Interestingly, some progress of soft sets has developed with graph theory. Ali et al. [19] introduced a new representation of graphs based on neighborhoods and soft sets. Akram and Nawaz presented the concepts of soft graphs [20,21] and fuzzy soft graphs [22]. Let $G=(X, \mathscr{E})$ be a simple graph; let $A$ be any nonempty set. Let $R \subseteq A \times X$ be an arbitrary relation from $A$ to $X$. A mapping $S$ from $A$ to $P(X)$ is denoted as $S: A \rightarrow$ $P(X)$ and defined as $S(x)=\{y \in X \mid x R y\}$ and a mapping $T$ from $A$ to $P(\mathscr{E})$ is denoted as $T: A \rightarrow P(\mathscr{E})$ and defined as $T(x)=\{u v \in \mathscr{E} \mid\{u, v\} \subseteq S(x)\}$. Then $(S, A)$ is a TiSS over $X$ and $(T, A)$ is a T1SS over $\mathscr{E}$. The notion of a soft graph is defined as follows.

Definition 2 (see [21]). A 4-tuple $G^{\circ}=(G, S, T, A)$ is called a soft graph if it satisfies the following conditions:

(i) $G=(X, \mathscr{E})$ is a simple graph.

(ii) $A$ is a nonempty set of parameters.

(iii) $[S, A]$ is a T1SS over $X$.

(iv) $[T, A]$ is a TiSS over $\mathscr{E}$.

(v) $(S(x), T(x))$, for all $x \in A$, represents a subgraph of $G$.

The soft graph $G^{\circ}=(G, S, T, A)$ can also be written as $G^{\circ}=\langle G, S, T\rangle=\{H(x) \mid x \in A\}$.

In the rest of the paper, soft graph will be written as type1 soft graph (briefly, T1SG). Some operations of T1SG are defined as follows.

Definition 3 (see [20]). Let $G^{\circ}=\left\langle S_{1}, T_{1}, A_{1}\right\rangle$ and $G^{\circ}=\left\langle S_{2}\right.$, $\left.\mathrm{T}_{2}, A_{2}\right\rangle$ be two TiSG of $G$. Then $G^{\prime \prime}$ is a type-1 soft subgraph of $G^{\circ}$ if

(i) $A_{2} \subseteq A_{1}$,

(ii) for each $x \in A_{2}, H_{2}(x)$ is a subgraph of $H_{1}(x)$.

Definition 4 (see [20]). Let $G_{1}^{\circ}=\left\langle S_{1}, T_{1}, A_{1}\right\rangle$ and $G_{2}^{\circ}=$ $\left\langle S_{2}, T_{2}, A_{2}\right\rangle$ be two T1SG of $G$. The extended union of $G_{1}^{\circ}$ and $G_{2}^{\circ}$, denoted by $G_{1}^{\circ} \sqcup_{\varepsilon} G_{2}^{\circ}=G^{\circ}=\langle I, J, C\rangle$, where $C=A_{1} \cup A_{2}$, is defined, $\forall \alpha \in C$, as

$$
\begin{aligned}
& I(\alpha)= \begin{cases}S_{1}(\alpha), & \text { if } \alpha \in A_{1}-A_{2} \\
S_{2}(\alpha), & \text { if } \alpha \in A_{2}-A_{1} \\
S_{1}(\alpha) \cup S_{2}(\alpha), & \text { if } \alpha \in A_{1} \cap A_{2}\end{cases} \\
& J(\alpha)= \begin{cases}T_{1}(\alpha), & \text { if } \alpha \in A_{1}-A_{2} \\
T_{2}(\alpha), & \text { if } \alpha \in A_{2}-A_{1} \\
T_{1}(\alpha) \cup T_{2}(\alpha), & \text { if } \alpha \in A_{1} \cap A_{2} .\end{cases}
\end{aligned}
$$
C\}.

It can be written as $G_{1}^{\circ} \sqcup_{\varepsilon} G_{2}^{\circ}=\{H(\alpha)=(I(\alpha), J(\alpha)) \mid \alpha \epsilon$

Definition 5 (see [20]). Let $G_{1}^{\circ}=\left\langle S_{1}, T_{1}, A_{1}\right\rangle$ and $G_{2}^{\circ}=$ $\left\langle S_{2}, T_{2}, A_{2}\right\rangle$ be two T1SG in $G$. The restricted intersection of $G_{1}^{\circ}=\left\langle S_{1}, T_{1}, A_{1}\right\rangle$ and $G_{2}^{\circ}=\left\langle S_{2}, T_{2}, A_{2}\right\rangle$, denoted by $G_{1}^{\circ} \Pi_{r} G_{2}^{\circ}=G^{\circ}=\langle I, J, C\rangle$, where $C=A_{1} \cap A_{2}$, is defined, $\forall \alpha \in C$, as $I(\alpha)=S_{1}(\alpha) \cap S_{2}(\alpha), J(\alpha)=T_{1}(\alpha) \cap T_{2}(\alpha)$.

It can be written as $G_{1}^{\circ} \Pi_{r} G_{2}^{\circ}=\{H(x)=(I(x), J(x)) \mid x \in$ C\}.

Definition 6 (see [20]). Let $G_{1}^{\circ}=\left\langle S_{1}, T_{1}, A_{1}\right\rangle$ and $G_{2}^{\circ}=$ $\left\langle S_{2}, T_{2}, A_{2}\right\rangle$ be two T1SG in $G$. The $A N D$ operation of $G_{1}^{\circ}$ and $G_{2}^{\circ}$, denoted by $G_{1}^{\circ} \wedge G_{2}^{\circ}=G^{\circ}=\langle I, J, C\rangle$, where $C=A_{1} \times A_{2}$, is defined, $\forall(\alpha, \beta) \in A_{1} \times A_{2}$, as $I(\alpha, \beta)=S_{1}(\alpha) \cap S_{2}(\beta)$, $J(\alpha, \beta)=T_{1}(\alpha) \cap T_{2}(\beta)$.

Definition 7 (see [20]). Let $G_{1}^{\circ}=\left\langle S_{1}, T_{1}, A_{1}\right\rangle$ and $G_{2}^{\circ}=$ $\left\langle S_{2}, T_{2}, A_{2}\right\rangle$ be two T1SG in $G$. The $O R$ operation of $G_{1}^{\circ}$ and $G_{2}^{\circ}$, denoted by $G_{1}^{\circ} \vee G_{2}^{\circ}=G^{\circ}=\langle I, J, C\rangle$, where $C=A_{1} \times A_{2}$, is defined, $\forall(\alpha, \beta) \in A_{1} \times A_{2}$, as $I(\alpha, \beta)=S_{1}(\alpha) \cup S_{2}(\beta)$, $J(\alpha, \beta)=T_{1}(\alpha) \cup T_{2}(\beta)$.

Definition 8 (see [20]). Let $G_{1}^{\circ}=\left\langle S_{1}, T_{1}, A_{1}\right\rangle$ and $G_{2}^{\circ}=$ $\left\langle S_{2}, T_{2}, A_{2}\right\rangle$ be two T1SG of $G_{1}$ and $G_{2}$, respectively, such that $A_{1} \cap A_{2}=\emptyset$. The Cartesian product of $G_{1}^{\circ}$ and $G_{2}^{\circ}$ is denoted and defined by $G_{1}^{\circ} \rtimes G_{2}^{\circ}=G^{\circ}=\left\langle K, A_{1} \times A_{2}\right\rangle$, $\forall(\alpha, \beta) \in A_{1} \times A_{2}, K(\alpha, \beta)=H_{1}(\alpha) \times H_{2}(\beta)$, where $H_{1}(\alpha)$ and $H_{2}(\beta)$ are the subgroups of $G_{1}$ and $G_{2}$, respectively.

Note that $K(\alpha, \beta)=H_{1}(\alpha) \times H_{2}(\beta)$ denotes the Cartesian product of two crisp subgraphs. That is, $G_{1}^{\circ} \rtimes G_{2}^{\circ}=\{K(\alpha, \beta) \mid$ $\left.(\alpha, \beta) \in A_{1} \times A_{2}\right\}$.

For basic definitions of graph and soft graph, see [20, 21, 23-25].

In order to deal with associations between parameters, Chatterjeea et al. [26] propose the concept of type-2 soft set which is a generalization of Molodtsov's soft set. It involves parameterization over an already parameterized set and hence has more freedom and efficiency compared to usual soft sets (termed as type-1 soft sets) in handling impreciseness. In fact, type-2 soft sets are a new approach to managing uncertainty. Interestingly, Chatterjeea et al. [26] investigate some basic operations on type- 2 soft sets and use the model of type- 2 soft sets in decision making problems. Recently, distance, entropy, and similarity measures of type- 2 soft sets are introduced by Chatterjeea et al. [27].

Let $X$ be universe set and let $E$ be the set of parameters.

Definition 9 (see [26]). Let $(X, E)$ be a soft universe and let $\sigma(X)$ be the collection of all TiSS over $(X, E)$. Then a mapping $S: A \rightarrow \sigma(X), A \subseteq E$ is called a type-2 soft set (briefly T2SS) over $(X, E)$ and it is denoted by $\left[S^{*}, A\right]$. In this case, corresponding to each parameter $\alpha \in A, S^{*}(\alpha)$ is a TiSS. Thus, for each $\alpha \in A$, there exists a T1SS, $\left(S_{\alpha}, L_{\alpha}\right)$ such that $S^{*}(\alpha)=\left(S_{\alpha}, L_{\alpha}\right)$, where $S_{\alpha}: L_{\alpha} \rightarrow P(X)$ and $L_{\alpha} \subset E$. In this case, we refer to the parameter set $A$ as the "primary set of parameters," while the set of parameters $\cup L_{\alpha}$ is known as the "underlying set of parameters."

In this study, we present an application of type-2 soft set in graph theory. We introduce vertex-neighbors based type- 2 
soft sets over $X$ (set of all vertices of a graph) and $\mathscr{E}$ (set of all edges of a graph). Moreover, we introduce some type-2 soft operations in graphs by presenting several examples to demonstrate these new concepts. We describe an application of type-2 soft graphs in communication networks and present procedure as an algorithm.

\section{Type-2 Soft Graphs}

The behavior and the selections of initial parameters and associated parameterized sets corresponding to initial parameters make the study of type- 2 soft sets very interesting. Motivated by this, we study type- 2 soft sets in graph theory and related properties. In a graph, every vertex regarding its location consists of a special relationship with its neighbors. Suppose that a company makes an analysis on the flow of transportation in two cities " $C_{1}$ " and " $C_{2}$ " of a country. We consider edge (line segment (link) between locations, that, is road) and vertex (location on the transportation network of interest (node), that is, town and road intersections). The company must make a survey on the flow of transportation from neighbor cities to control transportation in " $C_{1}$ " and " $\mathrm{C}_{2}$ " and obtain a graphical representation to analyze the flow of transportation. The vertex-neighbors correspondence has an essential role in the structure of a graph. The type- 2 soft set is also based on the correspondence of initial parameters and underlying parameters. The set of all neighbors of an element $x \in X$ is defined as follows.

Definition 10. Let $G=(X, \mathscr{E})$ be a simple graph. The set of neighbors of an element $x \in X$ is denoted by $N B_{x}$ and defined by $N B_{x}=\{z \in X \mid x z \in \mathscr{E}\}$.

Let $G=(X, \mathscr{E})$ be a simple graph and $A \subseteq X$. Then $N B_{A}=\bigcup_{x \in A} N B_{x}$. Let a subset $\mathscr{R}$ of $N B_{A} \times X$ be an arbitrary relation from $N B_{A}$ to $X$. The set of all T1SS over $X$ is denoted by $\Gamma(X)$ and the set of all T1SS over $\mathscr{E}$ is denoted by $\Gamma(\mathscr{E})$.

Definition 11. Let $G=(X, \mathscr{E})$ be a simple graph; let $A \subset X$ and $\Gamma(X)$ be the collection of all T1SS over $X$. Then a mapping $S^{*}$ : $A \rightarrow \Gamma(X)$ is called a T2SS over $X$ and it is denoted by $\left[S^{*}, A\right]$. In this case, corresponding to each vertex $x \in A, S^{*}(x)$ is a T1SS such that $S^{*}(x)=\left(S_{x}, N B_{x}\right)$, where $S_{x}: N B_{x} \rightarrow P(X)$ can be defined as $S_{x}(z)=\{y \in X \mid z \mathscr{R} y\}$, for all $z \in N B_{x} \subseteq$ $X$, and $N B_{x}$ is the set of all neighbors of $x \in A$. This T2SS is also called a vertex-neighbors induced type-2 soft set (briefly, VN-type-2 soft set) over $X$.

Definition 12. Let $G=(X, \mathscr{E})$ be a simple graph; let $A \subset X$ and $\Gamma(\mathscr{E})$ be the collection of all T1SS over $\mathscr{E}$. Let $\left[S^{*}, A\right]$ be a VN-type-2 soft set over $X$. Then a mapping $T^{*}: A \rightarrow \Gamma(\mathscr{E})$ is called a T2SS over $\mathscr{E}$ and it is denoted by $\left[T^{*}, A\right]$. In this case, corresponding to each vertex $x \in A, T^{*}(x)$ is a T1SS such that $T^{*}(x)=\left(T_{x}, N B_{\mathrm{x}}\right)$, where $T_{x}: N B_{x} \rightarrow P(\mathscr{E})$ can be defined as $T_{x}(z)=\left\{\alpha \beta \in \mathscr{E} \mid\{\alpha, \beta\} \subseteq S_{x}(z)\right\}$, for all $z \in N B_{x} \subseteq X$, and $N B_{x}$ is the set of all neighbors of $x \in A$. This T2SS is also called a VN-type-2 soft set over $\mathscr{E}$.

As it is mentioned in the above definitions, $S^{*}(x)=$ $\left(S_{x}, N B_{x}\right)$ and $T^{*}(x)=\left(T_{x}, N B_{x}\right) \forall x \in A$ are T1SS over $X$ and over $\mathscr{E}$, respectively. If $\left(S_{x}(z), T_{x}(z)\right) \forall z \in N B_{x}$

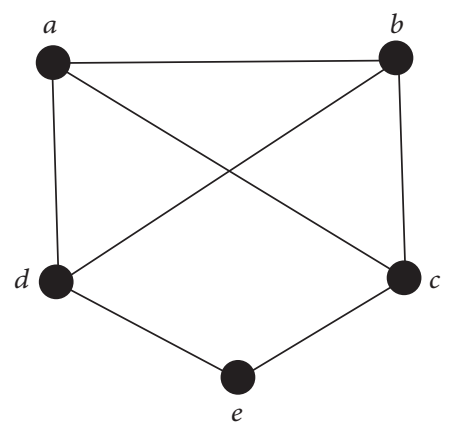

FIGURE 1: Undirected graph.

represents a subgraph of $G$, then by the definition of T1SG $\left(G, S_{x}, T_{x}, N B_{x}\right)$ will be a T1SG of $G$. This T1SG can also be represented by $\left\langle G, S_{x}, T_{x}\right\rangle=\left\{H_{x}(z) \mid z \in N B_{x}\right\}$.

2.1. Type-2 Soft Graph. A 5-tuple $\mathscr{G}^{*}=\left(G, S^{*}, T^{*}, A, N B_{A}\right)$ is called a type-2 soft graph (briefly, T2SG) if it satisfies the following conditions:

(i) $G=(X, \mathscr{E})$ is a simple graph.

(ii) $A$ is a nonempty set of parameters.

(iii) $\left[S^{*}, A\right]$ is a VN-type-2 soft set over $X$.

(iv) $\left[T^{*}, A\right]$ is a VN-type-2 soft set over $\mathscr{E}$.

(v) T1SS corresponding to $\left(S^{*}(x), T^{*}(x)\right) \forall x \in A$, represents a type-1 soft graph.

A T2SG can also be represented by $\mathscr{G}^{*}=\left\langle S^{*}, T^{*}, A\right\rangle=$ $\left\{H^{*}(x) \mid x \in A\right\}$, where $H^{*}(x)=\left(H_{x}, N B_{x}\right)$ such that $H_{x}(z)=\left(S_{x}(z), T_{x}(z)\right), \forall z \in N B_{x}$. The set of all T2SG of $G$ is denoted by $\mathscr{T} \mathcal{S} \mathscr{G}(G)$.

Although the above representation of graphs provides a new framework for the applications of graph theory, it is restricted to the information from neighbors. In this regard, a likeness between parameterized vertices appears in some cases such as distinction between T2SG $\left\langle S^{*}, T^{*}, A\right\rangle$ and $\left\langle S^{\prime *}, T^{\prime *}, A\right\rangle$ is limited toward respective T1SG. However, T2SG is more general and hence a more efficient approach in graph theory as compared to T1SG.

Definition 13. A T2SG $\mathscr{G}^{*}=\left(G, S^{*}, T^{*}, A, N B_{A}\right)$ is said to be vertex-induced if $H^{*}(\gamma)=\left(S^{*}(\gamma), T^{*}(\gamma)\right)=S^{*}(\gamma)$ for all $\gamma \in A$.

Definition 14. A T2SG $\mathscr{G}^{*}=\left(G, S^{*}, T^{*}, A, N B_{A}\right)$ is said to be edge-induced if $H^{*}(\gamma)=\left(S^{*}(\gamma), T^{*}(\gamma)\right)=T^{*}(\gamma)$ for all $\gamma \in A$.

Example 15. Consider a graph $G=(X, \mathscr{E})$ as shown in Figure 1.

Let $A=\{a, b\} \subset X$. Then $N B_{a}=\{b, c, d\}, N B_{b}=$ $\{a, c, d\}$. Let $\left[S^{*}, A\right]$ and $\left[T^{*}, A\right]$ be two T2SS over $X$ and $\mathscr{E}$, respectively, such that $S^{*}(x)=\left(S_{x}, N B_{x}\right)$ and $T^{*}(x)=$ $\left(T_{x}, N B_{x}\right)$ for all $x \in A$. Let $S_{a}(\alpha)=\{y \in X \mid \alpha \mathscr{R} y \Leftrightarrow$ $d(\alpha, y) \leq 1\}, T_{a}(\alpha)=\left\{u v \in \mathscr{E} \mid\{u, v\} \subseteq S_{a}(\alpha)\right\} \forall \alpha \in N B_{a}$ 

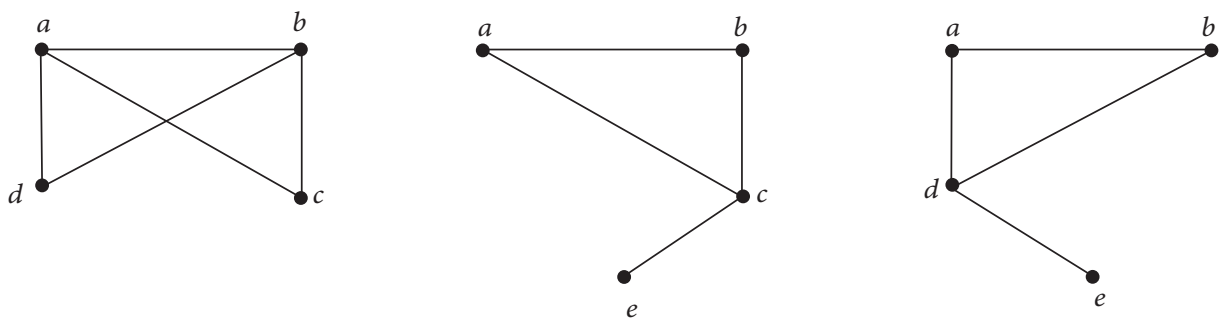

$$
H_{a}(b)=\left(S_{a}(b), T_{a}(b)\right)
$$

$H_{a}(c)=\left(S_{a}(c), T_{a}(c)\right)$

$H_{a}(d)=\left(S_{a}(d), T_{a}(d)\right)$
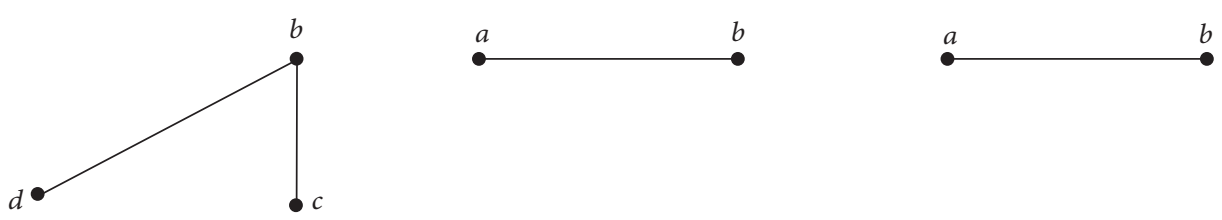

$H_{b}(a)=\left(S_{b}(a), T_{b}(a)\right)$

$H_{b}(c)=\left(S_{b}(c), T_{b}(c)\right)$

$H_{b}(d)=\left(S_{b}(d), T_{b}(d)\right)$

Figure 2: $G^{*}=\left(H^{*}(a), H^{*}(b)\right)$.

and $S_{b}(\beta)=\left\{y^{\prime} \in X \mid \beta \mathscr{R} y^{\prime} \Leftrightarrow d\left(\beta, y^{\prime}\right)=1\right\}, T_{b}(\beta)=$ $\left\{u^{\prime} v^{\prime} \in \mathscr{E} \mid\left\{u^{\prime}, v^{\prime}\right\} \subseteq S_{b}(\beta)\right\} \forall \beta \in N B_{b}$. Then

$$
\begin{aligned}
S^{*}(a) & =\left\{\frac{b}{\{a, b, d, c\}}, \frac{c}{\{a, b, c, e\}}, \frac{d}{\{a, b, d, e\}}\right\}, \\
T^{*}(a) & =\left\{\frac{b}{\{a b, a d, a c, b c, b d\}}, \frac{c}{\{a b, a c, c e, b c\}},\right. \\
& \left.\frac{d}{\{a b, a d, d e, d b\}}\right\}, \\
S^{*}(b) & =\left\{\frac{a}{\{b, c, d\}}, \frac{c}{\{a, b, e\}}, \frac{d}{\{a, b, e\}}\right\}, \\
T^{*}(b) & =\left\{\frac{a}{\{b c, b d\}}, \frac{c}{\{a b\}}, \frac{d}{\{a b\}}\right\} .
\end{aligned}
$$

Figure 2 shows the respective T1SG corresponding to $H^{*}(a)=\left(S^{*}(a), T^{*}(a)\right)$ and $H^{*}(b)=\left(S^{*}(b), T^{*}(b)\right)$, respectively. Hence, $\mathscr{G}^{*}=\left(H^{*}(a), H^{*}(b)\right)$ is a T2SG of $G$. It is also called VN-type-2 soft graph.

Example 16. Consider a crisp graph $G=(X, \mathscr{E})$ such that $X=$ $\{a, b, c, u, v\}$ and $\mathscr{E}=\{a b, c u, c b, a v, u v\}$. Let $A=\left\{e_{1}, e_{2}, e_{3}\right\}$ be a nonempty set of parameters and $N B_{e_{1}}=\left\{e_{2}, e_{4}\right\}, N B_{e_{2}}=$ $\left\{e_{1}, e_{6}\right\}$, and $N B_{e_{3}}=\left\{e_{4}, e_{5}\right\}$. We may define the pair of T2SS $\left(\left[S^{*}, A\right],\left[T^{*}, A\right]\right)$ as follows:

$$
\begin{aligned}
H^{*} & \left(e_{1}\right)=\left(S^{*}\left(e_{1}\right), T^{*}\left(e_{1}\right)\right) \\
= & \left(\left\{\frac{e_{2}}{\{a, v\}}, \frac{e_{4}}{\{b, c\}}\right\},\left\{\frac{e_{2}}{\{a v\}}, \frac{e_{4}}{\{b c\}}\right\}\right),
\end{aligned}
$$

$$
\begin{aligned}
H^{*} & \left(e_{2}\right)=\left(S^{*}\left(e_{2}\right), T^{*}\left(e_{2}\right)\right) \\
= & \left(\left\{\frac{e_{1}}{\{a, v, b\}}, \frac{e_{6}}{\{a, v, u\}}\right\},\left\{\frac{e_{1}}{\{a v, a b\}}, \frac{e_{6}}{\{a v, v u\}}\right\}\right), \\
H^{*} & \left(e_{3}\right)=\left(S^{*}\left(e_{3}\right), T^{*}\left(e_{3}\right)\right) \\
\quad= & \left(\left\{\frac{e_{4}}{\{b, c, u\}}, \frac{e_{5}}{\{v, u\}}\right\},\left\{\frac{e_{4}}{\{b c, c u\}}, \frac{e_{5}}{\{v u\}}\right\}\right) .
\end{aligned}
$$

Then the subgraphs of $G$ corresponding to parameters (vertices) $e_{1}, e_{2}$, and $e_{3}$ are shown in Figure 3 . Hence, $\mathscr{G}^{*}=$ $\left(H^{*}\left(e_{1}\right), H^{*}\left(e_{2}\right), H^{*}\left(e_{3}\right)\right)$ is a T2SG of $G$. Consider $\mathscr{P}=$ $\bigcup_{x \in A} N B_{x}=\left\{e_{1}, e_{2}, e_{4}, e_{5}, e_{6}\right\}$. We may symbolize $\alpha \in N B_{x}$, as $(x \mapsto \alpha)$ and denote a set of associations of $A$, as $(A \mapsto$ $\mathscr{P})=\left\{(x \mapsto \alpha) \mid \alpha \in N B_{x}\right\}$. Then tabular representation of T2SG is given in Table 1.

\section{Operations on Type-2 Soft Graphs}

In this section, we present type-2 soft subgraph of a T2SG, union, intersection, OR operation, AND operation, and Cartesian product of T2SG.

Definition 17. Let $\mathscr{G}_{1}^{*}=\left\langle S_{1}^{*}, T_{1}^{*}, A_{1}\right\rangle$ and $\mathscr{G}_{2}^{*}=\left\langle S_{2}^{*}, T_{2}^{*}, A_{2}\right\rangle$ be two T2SG of $G$. Then $\mathscr{G}_{2}^{*}$ is a type-2 soft subgraph of $\mathscr{G}_{1}^{*}$ if

(i) $A_{2} \subseteq A_{1}$,

(ii) for each $x \in A_{2}$, T1SG corresponding to $H_{2}^{*}(x)=$ $\left(S_{2}^{*}(x), T_{2}^{*}(x)\right)$ is a type-1 soft subgraph of T1SG corresponding to $H_{1}^{*}(x)=\left(S_{1}^{*}(x), T_{1}^{*}(x)\right)$. 
TABLE 1: Tabular representation of a type-2 soft graph.

\begin{tabular}{|c|c|c|c|c|c|c|c|c|c|c|}
\hline \multirow{2}{*}{$(A \nrightarrow \mathscr{P})$} & \multicolumn{5}{|c|}{$X$} & \multicolumn{5}{|c|}{$\mathscr{E}$} \\
\hline & $a$ & $b$ & $c$ & $u$ & $v$ & $a b$ & $c u$ & $c b$ & $a v$ & $u v$ \\
\hline$e_{1} \mapsto e_{2}$ & 1 & 0 & 0 & 0 & 1 & 0 & 0 & 0 & 1 & 0 \\
\hline$e_{1} \longmapsto e_{4}$ & 0 & 1 & 1 & 1 & 0 & 0 & 1 & 1 & 0 & 0 \\
\hline$e_{2} \longmapsto e_{1}$ & 1 & 1 & 0 & 0 & 1 & 1 & 0 & 0 & 1 & 0 \\
\hline$e_{2} \longmapsto e_{6}$ & 1 & 0 & 0 & 1 & 1 & 0 & 0 & 0 & 1 & 1 \\
\hline$e_{3} \mapsto e_{4}$ & 0 & 1 & 1 & 1 & 0 & 0 & 1 & 1 & 0 & 0 \\
\hline$e_{3} \mapsto e_{5}$ & 0 & 0 & 0 & 1 & 1 & 0 & 0 & 0 & 0 & 1 \\
\hline
\end{tabular}
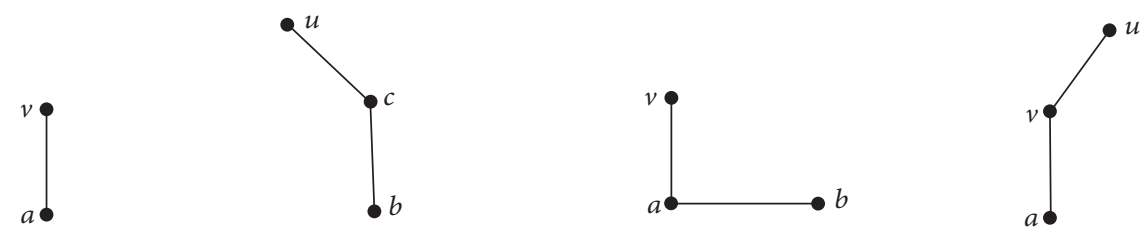

$\frac{H_{e_{1}}\left(e_{2}\right)=\left(S_{e_{1}}\left(e_{2}\right), T_{e_{1}}\left(e_{2}\right)\right) H_{e_{1}}\left(e_{4}\right)=\left(S_{e_{1}}\left(e_{4}\right), T_{e_{1}}\left(e_{4}\right)\right)}{H^{*}\left(e_{1}\right)=\left(S^{*}\left(e_{1}\right), T^{*}\left(e_{1}\right)\right)} \quad \frac{H_{e_{2}}\left(e_{1}\right)=\left(S_{e_{2}}\left(e_{1}\right), T_{e_{2}}\left(e_{1}\right)\right) H_{e_{2}}\left(e_{6}\right)=\left(S_{e_{2}}\left(e_{6}\right), T_{e_{2}}\left(e_{6}\right)\right)}{H^{*}\left(e_{2}\right)=\left(S^{*}\left(e_{2}\right), T^{*}\left(e_{2}\right)\right)}$
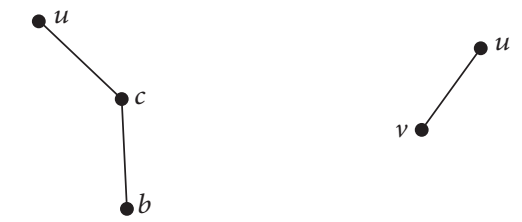

$\frac{H_{e_{3}}\left(e_{4}\right)=\left(S_{e_{3}}\left(e_{4}\right), T_{e_{3}}\left(e_{4}\right)\right) H_{e_{3}}\left(e_{5}\right)=\left(S_{e_{3}}\left(e_{5}\right), T_{e_{3}}\left(e_{5}\right)\right)}{H^{*}\left(e_{3}\right)=\left(S^{*}\left(e_{3}\right), T^{*}\left(e_{3}\right)\right)}$

Figure 3: $\mathscr{G}^{*}=\left(H^{*}\left(e_{1}\right), H^{*}\left(e_{2}\right), H^{*}\left(e_{3}\right)\right)$.

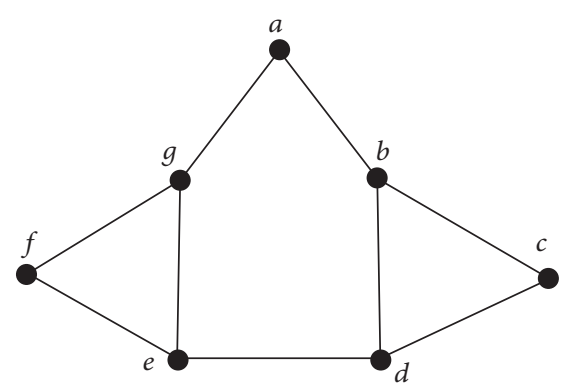

FIGURE 4: Undirected graph.

Example 18. Consider a graph $G=(X, \mathscr{E})$ as shown in Figure 4.

Let $A=\{a, c, f\}$ and $B=\{c, f\}$. Then $N B_{a}=\{g, b\}$, $N B_{c}=\{b, d\}$, and $N B_{f}=\{g, e\}$. Let $\left[S^{*}, A\right]$ and $\left[T^{*}, A\right]$ be two T2SS over $X$ and $\mathscr{E}$, respectively, such that $S^{*}(x)=$ $\left(S_{x}, N B_{x}\right)$ and $T^{*}(x)=\left(T_{x}, N B_{x}\right)$ for all $x \in A$, where $S_{x}(z)=\{y \in X \mid z \mathscr{R} y \Leftrightarrow 2 \leq d(z, y) \leq 3\}$ and $T_{x}(z)=\left\{\alpha \beta \in \mathscr{E} \mid\{\alpha, \beta\} \subseteq S_{x}(z)\right\}$ for all $z \in N B_{x} \subseteq X$. Now, T2SS $\left[S^{*}, A\right]$ and $\left[T^{*}, A\right]$ are as in the following:

$$
S^{*}(a)=\left\{\frac{b}{\{g, e, f\}}, \frac{g}{\{b, c, d\}}\right\}
$$

$$
\begin{aligned}
& T^{*}(a)=\left\{\frac{b}{\{e g, f e, g f\}}, \frac{g}{\{b c, c d, d b\}}\right\}, \\
& S^{*}(c)=\left\{\frac{b}{\{g, e, f\}}, \frac{d}{\{a, g, f\}}\right\}, \\
& T^{*}(c)=\left\{\frac{b}{\{e g, f d, g f\}}, \frac{d}{\{a g, g f\}}\right\}, \\
& S^{*}(f)=\left\{\frac{g}{\{b, c, d\}}, \frac{e}{\{a, b, c\}}\right\}, \\
& T^{*}(f)=\left\{\frac{g}{\{b c, c d, b d\}}, \frac{e}{\{a b, b c\}}\right\} .
\end{aligned}
$$

Thus, $\mathscr{G}^{*}=\left(H^{*}(a), H^{*}(c), H^{*}(f)\right)$ is a T2SG as shown in Figure 5.

Let $\left[S^{* *}, B\right]$ and $\left[T^{*}, B\right]$ be two T2SS over $X$ and $\mathscr{E}$, respectively, such that $S^{\prime *}(x)=\left(S_{x}^{\prime}, N B_{x}\right)$ and $T^{\prime *}(x)=$ $\left(T_{x}^{\prime}, N B_{x}\right)$ for all $x \in B$, where $S_{x}^{\prime}(z)=\{y \in X \mid z \mathscr{R} y \Leftrightarrow$ $d(z, y)=\operatorname{rad}(G)\}$, and $T_{x}^{\prime}(z)=\left\{\alpha \beta \in \mathscr{E} \mid\{\alpha, \beta\} \subseteq S_{x}^{\prime}(z)\right\}$ for all $z \in N B_{x} \subseteq X$. Then T2SS $\left[S^{\prime *}, B\right]$ and $\left[T^{*}, B\right]$ are as in the following:

$$
S^{\prime *}(c)=\left\{\frac{b}{\{g, e\}}, \frac{d}{\{a, g, f\}}\right\}
$$



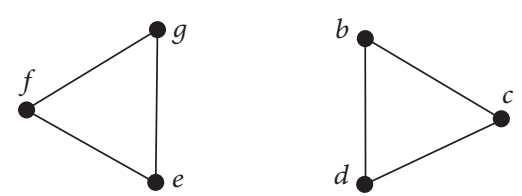

$\underline{H_{b}(g)=\left(S_{b}(g), T_{b}(g)\right) \quad H_{a}(g)=\left(S_{a}(g), T_{a}(g)\right)}$ $H^{*}(a)=\left(S^{*}(a), T^{*}(a)\right)$

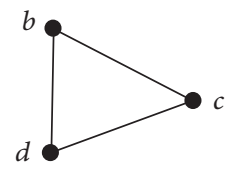

$$
\frac{H_{f}(g)=\left(S_{f}(g), T_{f}(g)\right) \quad H_{f}(e)=\left(S_{f}(e), T_{f}(e)\right)}{H^{*}(f)=\left(S^{*}(f), T^{*}(f)\right)}
$$

Figure 5: $\mathscr{G}^{*}=\left(H^{*}(a), H^{*}(c), H^{*}(f)\right)$.
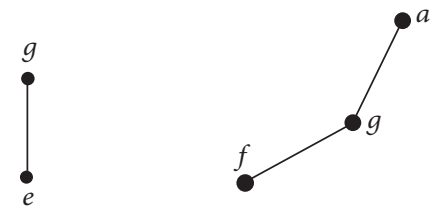

$\frac{H^{\prime}{ }_{c}(b)=\left(S^{\prime}{ }_{c}(b), T^{\prime}{ }_{c}(b)\right) \quad H^{\prime}{ }_{c}(d)=\left(S^{\prime}{ }_{c}(d), T^{\prime}{ }_{c}(d)\right)}{H^{\prime *}(c)=\left(S^{\prime *}(c), T^{\prime *}(c)\right)}$$$
\frac{H^{\prime}{ }_{f}(g)=\left(S^{\prime}{ }_{f}(g), T^{\prime}{ }_{f}(g)\right) \quad H^{\prime}(e)=\left(S^{\prime}{ }_{f}(e), T^{\prime}{ }_{f}(e)\right)}{H^{\prime *}(f)=\left(S^{\prime *}(f), T^{\prime *}(f)\right)}
$$

Figure 6: $\mathscr{G}^{\prime *}=\left(H^{\prime *}(c), H^{\prime *}(f)\right)$.

$$
\begin{aligned}
T^{\prime *}(c) & =\left\{\frac{b}{\{g e\}}, \frac{d}{\{a g, g f\}}\right\}, \\
S^{\prime *}(f) & =\left\{\frac{g}{\{d, b\}}, \frac{e}{\{a, b, c\}}\right\}, \\
T^{\prime *}(f) & =\left\{\frac{g}{\{b d\}}, \frac{e}{\{a b, b c\}}\right\} .
\end{aligned}
$$

Therefore, $\mathscr{G}^{\prime *}=\left(H^{\prime *}(c), H^{\prime *}(f)\right)$ is a T2SG of $G$ as shown in Figure 6.

One can easily check that $\mathscr{G}^{\prime *}=\left(H^{\prime *}(c), H^{\prime *}(f)\right)$ is a type-2 soft subgraph of $\mathscr{G}^{*}=\left(H^{*}(a), H^{*}(c), H^{*}(f)\right)$.

Theorem 19. Let $\mathscr{G}_{1}^{*}=\left\langle S_{1}^{*}, T_{1}^{*}, A_{1}\right\rangle$ and $\mathscr{G}_{2}^{*}=\left\langle S_{2}^{*}, T_{2}^{*}, A_{2}\right\rangle$ be two T2SG of $G$. Then $\mathscr{G}_{2}^{*}$ is a type-2 soft subgraph of $\mathscr{G}_{1}^{*}$ if and only if $S_{2}^{*}(x) \widetilde{\subseteq} S_{1}^{*}(x)$ and $T_{2}^{*}(x) \widetilde{\subseteq} T_{1}^{*}(x)$ for all $x \in A_{2}$.

Proof. Suppose $\mathscr{G}_{2}^{*}$ is a T2SG of $\mathscr{G}_{1}^{*}$. Then by the definition of T2SG,

(i) $A_{2} \subseteq A_{1}$,

(ii) for each $x \in A_{2}$, T1SG corresponding to $H_{2}^{*}(x)=$ $\left(S_{2}^{*}(x), T_{2}^{*}(x)\right)$ is a type-1 soft subgraph of T1SG corresponding to $H_{1}^{*}(x)=\left(S_{1}^{*}(x), T_{1}^{*}(x)\right)$.

Since T1SG corresponding to $H_{2}^{*}(x)$ is a type-1 soft subgraph of T1SG corresponding to $H_{1}^{*}(x)$ for all $x \in A_{2}$. Then $S_{2}^{*}(x) \widetilde{\subseteq} S_{1}^{*}(x)$ and $T_{2}^{*}(x) \widetilde{\subseteq} T_{1}^{*}(x)$ for all $x \in A_{2}$.
Conversely, $S_{2}^{*}(x) \widetilde{\subseteq} S_{1}^{*}(x)$ and $T_{2}^{*}(x) \widetilde{\subseteq} T_{1}^{*}(x)$ for all $x \in$ $A_{2}$. As $\mathscr{G}_{1}^{*}$ is a T2SG of $G$, T1SS corresponding to $H_{1}^{*}(x)=$ $\left(S_{1}^{*}(x), T_{1}^{*}(x)\right)$ is a T1SG of $G$ for all $x \in A_{1}$. Also, $\mathscr{G}_{2}^{*}$ is a T2SG of $G$; T1SS corresponding to $H_{2}^{*}(x)=\left(S_{2}^{*}(x), T_{2}^{*}(x)\right)$ is a T1SG of $G$ for all $x \in A_{2}$. This implies that T1SG corresponding to $H_{2}^{*}(x)$ is a type-1 soft subgraph of T1SG corresponding to $H_{1}^{*}(x)$ for all $x \in A_{2}$. Thus, $\mathscr{G}_{2}^{*}$ is a type-2 soft subgraph of $\mathscr{G}_{1}^{*}$.

Definition 20. Let $\mathscr{G}_{1}^{*}=\left\langle S_{1}^{*}, T_{1}^{*}, A_{1}\right\rangle$ and $\mathscr{G}_{2}^{*}=\left\langle S_{2}^{*}, T_{2}^{*}, A_{2}\right\rangle$ be two T2SG of $G$. The union of $\mathscr{G}_{1}^{*}$ and $\mathscr{G}_{2}^{*}$, denoted by $\mathscr{G}_{1}^{*} \sqcup$ $\mathscr{G}_{2}^{*}=\mathscr{G}^{*}=\left\langle I^{*}, J^{*}, C\right\rangle$, where $C=A_{1} \cup A_{2}$, is defined, $\forall \alpha \in C$, as

$$
I^{*}(\alpha)= \begin{cases}S_{1}^{*}(\alpha), & \text { if } \alpha \in A_{1}-A_{2} \\ S_{2}^{*}(\alpha), & \text { if } \alpha \in A_{2}-A_{1} \\ S_{1}^{*}(\alpha) \widetilde{\cup} S_{2}^{*}(\alpha), & \text { if } \alpha \in A_{1} \cap A_{2},\end{cases}
$$

where $S_{1}^{*}(\alpha) \widetilde{\cup} S_{2}^{*}(\alpha)$ for all $\alpha \in A_{1} \cap A_{2}$ refers to the usual type-1 soft union between the respective T1SS corresponding to $S_{1}^{*}(\alpha)$ and $S_{2}^{*}(\alpha)$, respectively. And,

$$
J^{*}(\alpha)= \begin{cases}T_{1}^{*}(\alpha), & \text { if } \alpha \in A_{1}-A_{2} \\ T_{2}^{*}(\alpha), & \text { if } \alpha \in A_{2}-A_{1} \\ T_{1}^{*}(\alpha) \tilde{\cup} T_{2}^{*}(\alpha), & \text { if } \alpha \in A_{1} \cap A_{2},\end{cases}
$$




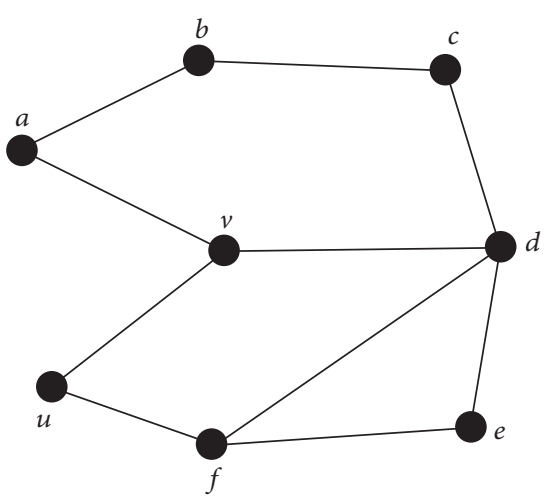

FIgURE 7: Simple graph.

where $T_{1}^{*}(\alpha) \widetilde{\cup} T_{2}^{*}(\alpha)$ for all $\alpha \in A_{1} \cap A_{2}$ refers to the usual type-1 soft union between the respective T1SS corresponding to $T_{1}^{*}(\alpha)$ and $T_{2}^{*}(\alpha)$, respectively.

It can be written as $\mathscr{G}_{1}^{*} \sqcup \mathscr{G}_{2}^{*}=\left\{H^{*}(\alpha)=\left(I^{*}(\alpha), J^{*}(\alpha)\right) \mid\right.$ $\alpha \in C\}$.

Example 21. Consider a graph $G=(X, \mathscr{E})$ as shown in Figure 7

Let $A=\{a, e\}$ and $B=\{b, e\}$. Then $N B_{a}=\{v, b\}, N B_{b}=$ $\{a, c\}$, and $N B_{e}=\{f, d\}$. Let $\left[S^{*}, A\right]$ and $\left[T^{*}, A\right]$ be two T2SS over $X$ and $\mathscr{E}$, respectively, such that $S^{*}(x)=\left(S_{x}, N B_{x}\right)$ and $T^{*}(x)=\left(T_{x}, N B_{x}\right)$ for all $x \in A$, where $S_{x}(z)=\{y \in X \mid$ $z \mathscr{R} y \Leftrightarrow d(z, y) \leq 1\}$ and $T_{x}(z)=\left\{\alpha \beta \in \mathscr{E} \mid\{\alpha, \beta\} \subseteq S_{x}(z)\right\}$ for all $z \in N B_{x} \subseteq X$. Now, T2SS $\left[S^{*}, A\right]$ and $\left[T^{*}, A\right]$ are as in the following:

$$
\begin{aligned}
S^{*}(a) & =\left\{\frac{b}{\{a, b, c\}}, \frac{v}{\{a, d, v, u\}}\right\}, \\
T^{*}(a) & =\left\{\frac{b}{\{a b, b c\}}, \frac{v}{\{v a, v d, v u\}}\right\}, \\
S^{*}(e) & =\left\{\frac{f}{\{d, e, f, u\}}, \frac{d}{\{d, e, f, v, c\}}\right\}, \\
T^{*}(e) & =\left\{\frac{f}{\{f u, f d, f e, d e\}}, \frac{d}{\{d e, d f, f e, d v\}}\right\} .
\end{aligned}
$$

Thus, $\mathscr{G}^{*}=\left(H^{*}(a), H^{*}(e)\right)$ is a T2SG of $G$.

Let $\left[S^{\prime *}, B\right]$ and $\left[T^{\prime *}, B\right]$ be two T2SS over $X$ and $\mathscr{E}$, respectively, such that $S^{\prime *}(x)=\left(S_{x}^{\prime}, N B_{x}\right)$ and $T^{\prime *}(x)=$ $\left(T_{x}^{\prime}, N B_{x}\right)$ for all $x \in B$, where $S_{x}^{\prime}(z)=\{y \in X \mid z \mathscr{R} y \Leftrightarrow$ $1 \leq d(z, y) \leq \operatorname{rad}(G)\}$, and $T_{x}^{\prime}(z)=\left\{\alpha \beta \in \mathscr{E} \mid\{\alpha, \beta\} \subseteq S_{x}^{\prime}(z)\right\}$ for all $z \in N B_{x} \subseteq X$. Then T2SS $\left[S^{\prime *}, B\right]$ and $\left[T^{\prime *}, B\right]$ are as in the following:

$$
\begin{aligned}
S^{\prime *}(b) & =\left\{\frac{a}{\{v, u, d, b, c\}}, \frac{c}{\{d, f, e, v, b, a\}}\right\}, \\
T^{\prime *}(b) & =\left\{\frac{a}{\{v u, d v, d c, c b\}}, \frac{c}{\{d f, e f, e d, v d, a b, a v\}}\right\},
\end{aligned}
$$

$$
\begin{aligned}
S^{\prime *}(e) & =\left\{\frac{f}{\{e, d, u, v, c\}}, \frac{d}{\{e, f, u, v, a, c, b\}}\right\}, \\
T^{\prime *}(e) & =\left\{\frac{f}{\{e d, d v, d c, u v\}}, \frac{d}{\{e f, u v, f u, v a, c b, a b\}}\right\} .
\end{aligned}
$$

Therefore, $\mathscr{G}^{\prime *}=\left(H^{\prime *}(b), H^{\prime *}(e)\right)$ is a T2SG of $G$.

Then union of $\mathscr{G}^{*}=\left\langle S^{*}, T^{*}, B\right\rangle$ and $\mathscr{G}^{\prime *}=\left\langle S^{\prime *}, T^{\prime *}, B\right\rangle$ is $\mathscr{G}^{*} \widetilde{\cup} \mathscr{G}^{*}=\left\langle I^{*}, J^{*}, C\right\rangle$, where $C=A \cup B$ and

$$
\begin{aligned}
& I^{*}(a)=\left\{\frac{b}{\{a, b, c\}}, \frac{v}{\{a, d, v, u\}}\right\}, \\
& J^{*}(a)=\left\{\frac{b}{\{a b, b c\}}, \frac{v}{\{v a, v d, v u\}}\right\}, \\
& I^{*}(b)=\left\{\frac{a}{\{v, u, d, b, c\}}, \frac{c}{\{d, f, e, v, b, a\}}\right\}, \\
& J^{*}(b)=\left\{\frac{a}{\{u v, d v, d c, c b\}}, \frac{c}{\{d f, e f, e d, v d, a b, a v\}}\right\}, \\
& I^{*}(e)=\left\{\frac{f}{\{e, f, u, d, v, c\}}, \frac{d}{X}\right\}, \\
& J^{*}(e)=\left\{\frac{f}{\{e d, d v, d c, u v, f e, f d, f u\}}, \frac{d}{E}\right\} .
\end{aligned}
$$

The union of $\mathscr{G}^{*}$ and $\mathscr{G}^{\prime *}$ is shown in Figure 8.

Note that, in the definition of T2SG, subgraphs in T1SG corresponding to $H^{*}(x)$ may be connected or disconnected subgraphs of a simple graph. However, in this section we are now focusing only on connected subgraphs of a simple graph.

Theorem 22. Let $\mathscr{G}_{1}^{*}=\left\langle S^{*}, T^{*}, A_{1}\right\rangle$ and $\mathscr{G}_{2}^{*}=\left\langle S^{\prime *}, T^{\prime *}, A_{2}\right\rangle$ be two T2SG of $G$ with $A_{1} \cap A_{2} \neq \emptyset$. Let T1SS corresponding to $S^{*}(\gamma)$ and $S^{*}(\eta)$ be $\left(S_{\gamma}, N B_{\gamma}\right)$ and $\left(S_{\eta}^{\prime}, N B_{\eta}\right)$, respectively. If for each $\alpha \in A_{1} \cap A_{2}, S_{\alpha}(x) \cap S_{\alpha}^{\prime}(x) \neq \emptyset \forall x \in N B_{\alpha}$, then the union of $\mathscr{G}_{1}^{*}$ and $\mathscr{G}_{2}^{*}$ is a T2SG of $G$.

Proof. The union of $\mathscr{G}_{1}^{*}=\left\langle S^{*}, T^{*}, A_{1}\right\rangle$ and $\mathscr{G}_{2}^{*}=\left\langle S^{\prime *}, T^{\prime *}\right.$, $\left.A_{2}\right\rangle$ is defined as $\mathscr{G}_{1}^{*} \sqcup \mathscr{G}_{2}^{*}=\mathscr{G}^{*}=\left\langle I^{*}, J^{*}, C\right\rangle$, where $C=$ $A_{1} \cup A_{2}$ for all $\alpha \in C$,

$$
I^{*}(\alpha)= \begin{cases}S^{*}(\alpha), & \text { if } \alpha \in A_{1}-A_{2} \\ S^{\prime *}(\alpha), & \text { if } \alpha \in A_{2}-A_{1} \\ S^{*}(\alpha) \widetilde{\cup} S^{\prime *}(\alpha), & \text { if } \alpha \in A_{1} \cap A_{2},\end{cases}
$$

where $S^{*}(\alpha) \widetilde{\cup} S^{\prime *}(\alpha)$ for all $\alpha \in A_{1} \cap A_{2}$ refers to the usual type-1 soft union between the respective T1SS corresponding to $S^{*}(\alpha)$ and $S^{\prime *}(\alpha)$, respectively. And,

$$
J^{*}(\alpha)= \begin{cases}T^{*}(\alpha), & \text { if } \alpha \in A_{1}-A_{2} \\ T^{\prime *}(\alpha), & \text { if } \alpha \in A_{2}-A_{1} \\ T^{*}(\alpha) \widetilde{\cup} T^{\prime *}(\alpha), & \text { if } \alpha \in A_{1} \cap A_{2},\end{cases}
$$



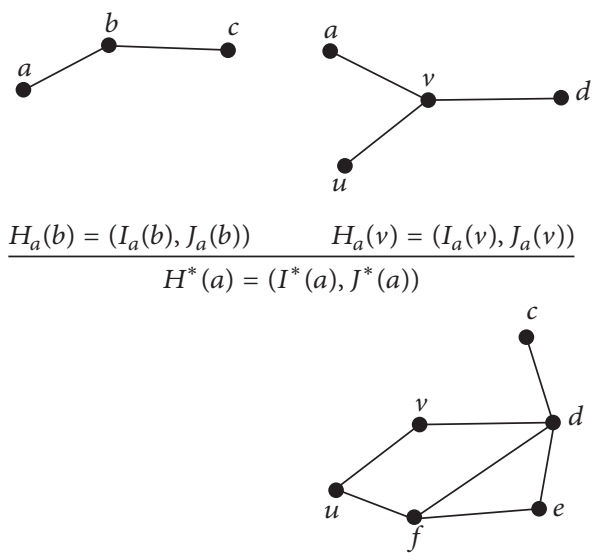

$H_{e}(f)=\left(I_{e}(f), J_{e}(f)\right)$
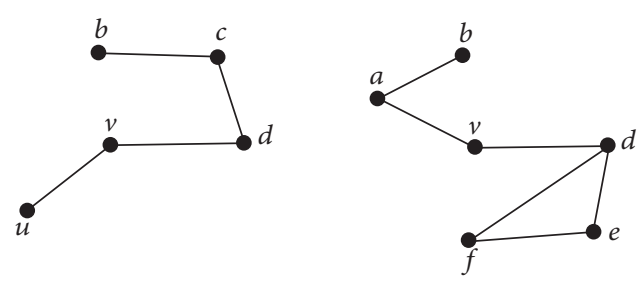

$\frac{H_{b}(a)=\left(I_{b}(a), J_{b}(a)\right) \quad H_{b}(c)=\left(I_{b}(c), J_{b}(c)\right)}{H^{*}(b)=\left(I^{*}(b), J^{*}(b)\right)}$

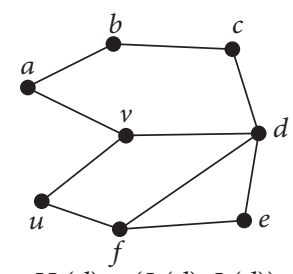

$H_{e}(d)=\left(I_{e}(d), J_{e}(d)\right)$

Figure 8: The union of $G^{*}$ and $G^{*}$.

where $T^{*}(\alpha) \widetilde{\cup} T^{\prime *}(\alpha)$ for all $\alpha \in A_{1} \cap A_{2}$ refers to the usual type-1 soft extended union between the respective T1SS corresponding to $T^{*}(\alpha)$ and $T^{* *}(\alpha)$, respectively.

$\mathscr{G}_{1}^{*}$ is connected T2SG of $G$. Then T1SS corresponding to $\left(S^{*}(\alpha), T^{*}(\alpha)\right)$ is a connected TiSG of $G$ for all $\alpha \in A_{1}-A_{2}$.

$\mathscr{G}_{2}^{*}$ is connected T2SG of $G$. Then T1SS corresponding to $\left(S^{\prime *}(\alpha), T^{\prime *}(\alpha)\right)$ is a connected T1SG of $G$ for all $\alpha \in A_{2}-A_{1}$.

Let $\alpha \in A_{1} \cap A_{2},\left(I^{*}(\alpha), J^{*}(\alpha)\right)=\left(S^{*}(\alpha) \widetilde{\cup} S^{\prime *}(\alpha)\right.$, $\left.T^{*}(\alpha) \widetilde{\cup} T^{\prime *}(\alpha)\right)$. Respective TiSS corresponding to $\left(S^{*}(\alpha)\right.$, $\left.T^{*}(\alpha)\right)$ and $\left(S^{\prime *}(\alpha), T^{\prime *}(\alpha)\right)$ are connected TISG of $G$. Given that $S_{\alpha}(x) \cap S_{\alpha}^{\prime}(x) \neq \emptyset \forall x \in N B_{\alpha}$, this implies that T1SS corresponding to $\left(I^{*}(\alpha), J^{*}(\alpha)\right)$ is connected TiSG of $G$. As $\alpha$ was taken to be an arbitrary element, theorem holds for all $\alpha \in A \cap B$. Thus, $\mathscr{G}_{1}^{*} \sqcup \mathscr{G}_{2}^{*}=\mathscr{G}^{*}=\left\langle I^{*}, J^{*}, A_{1} \cup A_{2}\right\rangle$ is a T2SG of $G$.

Lemma 23. Let $\mathscr{G}_{1}^{*}=\left\langle S_{1}^{*}, T_{1}^{*}, A_{1}\right\rangle$ and $\mathscr{G}_{2}^{*}=\left\langle S_{2}^{*}, T_{2}^{*}, A_{2}\right\rangle$ be two T2SG of G. If $A_{1} \cap A_{2}=\emptyset$, then their union is a T2SG of G.

Definition 24. Let $\mathscr{G}_{1}^{*}=\left\langle S_{1}^{*}, T_{1}^{*}, A_{1}\right\rangle$ and $\mathscr{G}_{2}^{*}=\left\langle S_{2}^{*}, T_{2}^{*}, A_{2}\right\rangle$ be two T2SG of $G$. The intersection of $\mathscr{G}_{1}^{*}$ and $\mathscr{G}_{2}^{*}$, denoted by $\mathscr{G}_{1}^{*} \sqcap \mathscr{G}_{2}^{*}=\mathscr{G}^{*}=\left\langle I^{*}, J^{*}, C\right\rangle$, where $C=A_{1} \cap A_{2}$, is defined by $I^{*}(\alpha)=S_{1}^{*}(\alpha) \widetilde{\cap} S_{2}^{*}(\alpha)$ and $J^{*}(\alpha)=T_{1}^{*}(\alpha) \widetilde{\cap} T_{2}^{*}(\alpha)$ for all $\alpha \in A_{1} \cap A_{2}$, where

(i) $S_{1}^{*}(\alpha) \widetilde{\cap} S_{2}^{*}(\alpha)$ for all $\alpha \in A_{1} \cap A_{2}$ refers to the usual type-1 soft intersection between the respective T1SS corresponding to $S_{1}^{*}(\alpha)$ and $S_{2}^{*}(\alpha)$, respectively,

(ii) $T_{1}^{*}(\alpha) \widetilde{\cap} T_{2}^{*}(\alpha)$ for all $\alpha \in A_{1} \cap A_{2}$ refers to the usual type-1 soft intersection between the respective TISS corresponding to $T_{1}^{*}(\alpha)$ and $T_{2}^{*}(\alpha)$, respectively. $\alpha \in C\}$.
Example 25. Consider T2SG $\mathscr{G}^{*}$ and $\mathscr{G}^{\prime *}$ defined in Example 18. The intersection between $\mathscr{G}^{*}$ and $\mathscr{G}^{\prime *}$ is equal to $\mathscr{G}^{\prime *}$ as shown in Figure 6.

Definition 26. Let $\mathscr{G}_{1}^{*}=\left\langle S_{1}^{*}, T_{1}^{*}, A_{1}\right\rangle$ and $\mathscr{G}_{2}^{*}=\left\langle S_{2}^{*}, T_{2}^{*}, A_{2}\right\rangle$ be two T2SG of $G$. The AND operation of $\mathscr{G}_{1}^{*}$ and $\mathscr{G}_{2}^{*}$, denoted by $\mathscr{G}_{1}^{*} \wedge \mathscr{G}_{2}^{*}=\mathscr{G}^{*}=\left\langle I^{*}, J^{*}, A \times B\right\rangle$ defined by $I^{*}(\gamma, \eta)=S_{1}^{*}(\gamma) \widetilde{\wedge} S_{2}^{*}(\eta), J^{*}(\gamma, \eta)=T_{1}^{*}(\gamma) \widetilde{\wedge} T_{2}^{*}(\eta)$ for all $(\gamma, \eta) \in A \times B$, where $S_{1}^{*}(\gamma) \widetilde{\wedge} S_{2}^{*}(\eta)$ for all $(\gamma, \eta) \in A \times B$, refers to the usual type-1 soft $A N D$ operation between the respective T1SS corresponding to $S_{1}^{*}(\gamma)$ and $S_{2}^{*}(\eta)$, respectively, and $T_{1}^{*}(\gamma) \widetilde{\wedge} T_{2}^{*}(\eta)$ for all $(\gamma, \eta) \in A \times B$ refers to the usual type-1 soft $A N D$ operation between the respective TiSS corresponding to $T_{1}^{*}(\gamma)$ and $T_{2}^{*}(\eta)$, respectively.

Example 27. Consider graph that is shown in Figure 7. Let $A=\{a, c\}$ and $B=\{b\}$. Then $N B_{a}=\{v, b\}, N B_{b}=\{a, c\}$, and $N B_{c}=\{b, d\}$. Let $\left[S^{*}, A\right]$ and $\left[T^{*}, A\right]$ be two T2SS over $X$ and $\mathscr{E}$, respectively, such that $S^{*}(x)=\left(S_{x}, N B_{x}\right)$ and $T^{*}(x)=$ $\left(T_{x}, N B_{x}\right)$ for all $x \in A$, where $S_{x}(z)=\{y \in X \mid z \mathscr{R} y \Leftrightarrow$ $d(z, y) \leq 1\}$ and $T_{x}(z)=\left\{\alpha \beta \in \mathscr{E} \mid\{\alpha, \beta\} \subseteq S_{x}(z)\right\}$ for all $z \in N B_{x} \subseteq X$. Now, T2SS $\left[S^{*}, A\right]$ and $\left[T^{*}, A\right]$ are as in the following:

$$
\begin{aligned}
S^{*}(a) & =\left\{\frac{b}{\{a, b, c\}}, \frac{v}{\{a, d, v, u\}}\right\}, \\
T^{*}(a) & =\left\{\frac{b}{\{a b, b c\}}, \frac{v}{\{v a, v d, v u\}}\right\}, \\
S^{*}(c) & =\left\{\frac{d}{\{d, e, f, v, c\}}, \frac{b}{\{a, b, c\}}\right\}, \\
T^{*}(c) & =\left\{\frac{d}{\{f e, f d, d e, d v, d c\}}, \frac{b}{\{a b, b c\}}\right\} .
\end{aligned}
$$

Then $\mathscr{G}^{*}=\left\langle S^{*}, T^{*}, A\right\rangle$ is T2SG of $G$.

Let $\left[S^{\prime *}, B\right]$ and $\left[T^{\prime *}, B\right]$ be two T2SS over $X$ and $\mathscr{E}$, respectively, such that $S^{\prime *}(x)=\left(S_{x}^{\prime}, N B_{x}\right)$ and $T^{\prime *}(x)=$ 


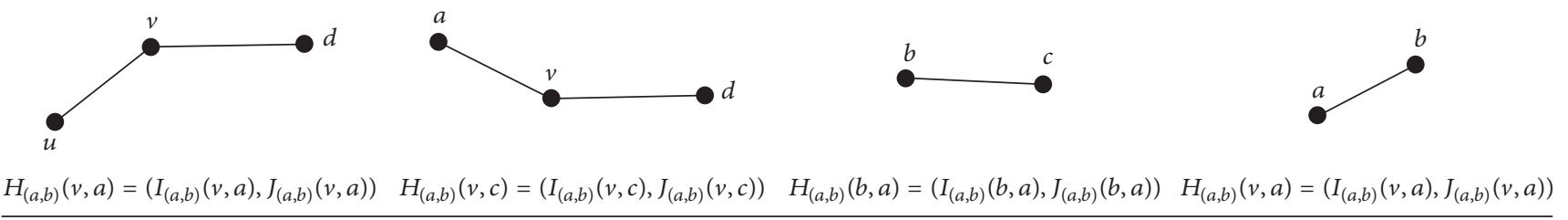

$H^{*}(a, b)=\left(I^{*}(a, b), J^{*}(a, b)\right)$

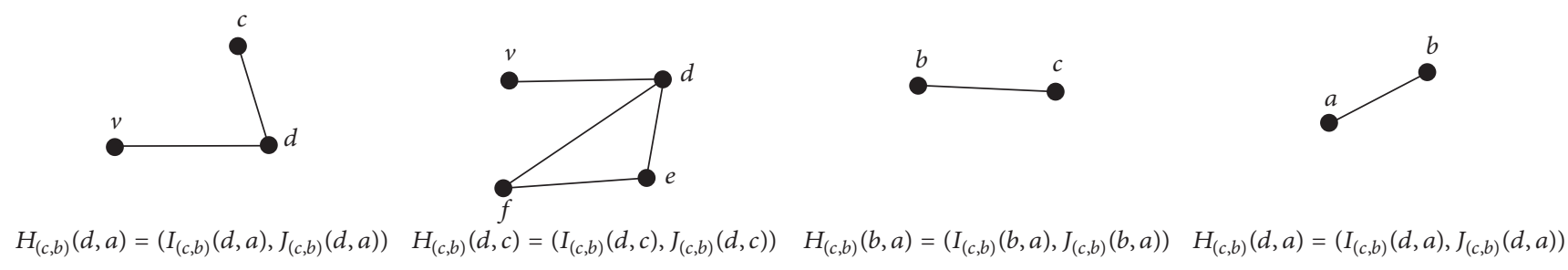

$H^{*}(c, b)=\left(I^{*}(c, b), J^{*}(c, b)\right)$

Figure 9: AND operations on $\mathscr{G}^{*}$ and $\mathscr{G}^{* \prime}$.

$\left(T_{x}^{\prime}, N B_{x}\right)$ for all $x \in B$, where $S_{x}^{\prime}(z)=\{y \in X \mid z \mathscr{R} y \Leftrightarrow$ $1 \leq d(z, y) \leq \operatorname{rad}(G)\}$ and $T_{x}^{\prime}(z)=\left\{\alpha \beta \in \mathscr{E} \mid\{\alpha, \beta\} \subseteq S_{x}^{\prime}(z)\right\}$ for all $z \in N B_{x} \subseteq X$. Then T2SS $\left[S^{\prime *}, B\right]$ and $\left[T^{\prime *}, B\right]$ are as in the following:

$$
\begin{aligned}
& S^{\prime *}(b)=\left\{\frac{a}{\{v, u, d, b, c\}}, \frac{c}{\{d, f, e, v, b, a\}}\right\}, \\
& T^{\prime *}(b)=\left\{\frac{a}{\{v u, d v, d c, c b\}}, \frac{c}{\{d f, e f, e d, v d, a b, a v\}}\right\} .
\end{aligned}
$$

Then $\mathscr{G}^{* \prime}=\left\langle S^{\prime *}, T^{\prime *}, B\right\rangle$ is T2SG of $G$.

The AND operation of $\mathscr{G}^{*}$ and $\mathscr{G}^{\prime *}$ is $\mathscr{G}^{*} \wedge \mathscr{G}^{\prime *}=\left\langle I^{*}, J^{*}\right.$, $A \times B\rangle$, where $A \times B=\{(a, b),(c, b)\}$ and

$$
\begin{aligned}
& I^{*}(a, b)=\left\{\frac{(v, a)}{\{v, u, d\}}, \frac{(v, c)}{\{a, v, d\}}, \frac{(b, a)}{\{b, c\}}, \frac{(b, c)}{\{b, a\}}\right\}, \\
& I^{*}(c, b)=\left\{\frac{(d, a)}{\{v, d, c\}}, \frac{(d, c)}{\{d, e, f, v\}}, \frac{(b, a)}{\{c, b\}}, \frac{(b, c)}{\{b, a\}}\right\}, \\
& J^{*}(a, b)=\left\{\frac{(v, a)}{\{v u, v d\}}, \frac{(v, c)}{\{a v, v d\}}, \frac{(b, a)}{\{b c\}}, \frac{(b, c)}{\{b a\}}\right\}, \\
& J^{*}(c, b)=\left\{\frac{(d, a)}{\{v d, d c\}}, \frac{(d, c)}{\{d f, e f, d e, d v\}}, \frac{(b, a)}{\{b c\}}, \frac{(b, c)}{\{b a\}}\right\} .
\end{aligned}
$$

The AND operation of $\mathscr{G}^{*}$ and $\mathscr{G}^{\prime *}$ is shown in Figure 9.

Theorem 28. Let $\mathscr{G}_{1}^{*}=\left\langle S^{*}, T^{*}, A_{1}\right\rangle$ and $\mathscr{G}_{2}^{*}=\left\langle S^{\prime *}, T^{* *}, A_{2}\right\rangle$ be two T2SG of G. Let T1SS corresponding to $S^{*}(\gamma)$ and $S^{* *}(\eta)$ be $\left(S_{\gamma}, N B_{\gamma}\right)$ and $\left(S_{\eta}^{\prime}, N B_{\eta}\right)$ for all $(\gamma, \eta) \in A_{1} \times A_{2}$, respectively, and let T1SS corresponding to $T^{*}(\gamma)$ and $T^{* *}(\eta)$ be $\left(T_{\gamma}, N B_{\gamma}\right)$ and $\left(T_{\eta}^{\prime}, N B_{\eta}\right)$ for all $(\gamma, \eta) \in A_{1} \times A_{2}$, respectively. For each $(\gamma, \eta) \in A_{1} \times A_{2}, S_{\gamma}(x) \cap S_{\eta}^{\prime}(y) \neq \emptyset$ and $T_{\gamma}(x) \cap T_{\eta}^{\prime}(y) \neq$ $\emptyset \forall(x, y) \in N B_{\gamma} \times N B_{\eta}$. Then $\mathscr{G}_{1}^{*} \wedge \mathscr{G}_{2}^{*}$ is a T2SG of $G$.

Proof. The AND operation of $\mathscr{G}_{1}^{*}$ and $\mathscr{G}_{2}^{*}$ is denoted by $\mathscr{G}_{1}^{*} \wedge$ $\mathscr{G}_{2}^{*}=\mathscr{G}^{*}=\left\langle I^{*}, J^{*}, A_{1} \times A_{2}\right\rangle$ and defined by $I^{*}(\gamma, \eta)=$
$S^{*}(\gamma) \tilde{\wedge} S^{*}(\eta)$ and $J^{*}(\gamma, \eta)=T^{*}(\gamma) \tilde{\wedge} T^{*}(\eta)$ for all $(\gamma, \eta) \in$ $A_{1} \times A_{2}$, where $S^{*}(\gamma) \widetilde{\wedge} S^{\prime *}(\eta)$ for all $(\gamma, \eta) \in A_{1} \times A_{2}$ refers to the usual type-1 soft $A N D$ operation between the respective T1SS corresponding to $S^{*}(\gamma)$ and $S^{* *}(\eta)$, respectively, and $T^{*}(\gamma) \tilde{\wedge} T^{* *}(\eta)$ for all $(\gamma, \eta) \in A_{1} \times A_{2}$ refers to the usual type-1 soft $A N D$ operation between the respective T1SS corresponding to $T^{*}(\gamma)$ and $T^{\prime *}(\eta)$, respectively.

$\mathscr{G}_{1}^{*}$ is connected T2SG of $G$. Then T1SS corresponding to $\left(S^{*}(\gamma), T^{*}(\gamma)\right)$ is a connected T1SG of $G$ for all $\gamma \in A_{1}$.

$\mathscr{G}_{2}^{*}$ is connected T2SG of $G$. Then T1SS corresponding to $\left(S^{\prime *}(\eta), T^{\prime *}(\eta)\right)$ is a connected T1SG of $G$ for all $\eta \in A_{2}$.

Let $(\gamma, \eta) \in A_{1} \times A_{2}$. It may be mentioned here that $I_{(\gamma, \eta)}(x, y)=S_{\gamma}(x) \cap S_{\eta}^{\prime}(y), J_{(\gamma, \eta)}(x, y)=T_{\gamma}(x) \cap T_{\eta}^{\prime}(y)$, such that $\left(I_{(\gamma, \eta)}(x, y), J_{(\gamma, \eta)}(x, y)\right)=\left(S_{\gamma}(x) \cap S_{\eta}^{\prime}(y), T_{\gamma}(x) \cap\right.$ $\left.T_{\eta}^{\prime}(y)\right) \forall(x, y) \in N B_{\gamma} \times N B_{\eta}$. Respective T1SS corresponding to $\left(S^{*}(\gamma), T^{*}(\eta)\right)$ and $\left(S^{\prime *}(\gamma), T^{\prime *}(\eta)\right)$ are connected T1SG of $G$. Given that $S_{\gamma}(x) \cap S_{\eta}^{\prime}(y) \neq \emptyset$ and $T_{\gamma}(x) \cap$ $T_{\eta}^{\prime}(y) \neq \emptyset \forall(x, y) \in N B_{\gamma} \times N B_{\eta}$, this implies that $\left(I_{(\gamma, \eta)}(x, y), J_{(\gamma, \eta)}(x, y)\right) \forall(x, y) \in N B_{\gamma} \times N B_{\eta}$ is a T1SG of $G$. Hence, T1SS corresponding to $\left(I^{*}(\gamma, \eta), J^{*}(\gamma, \eta)\right)$ is connected T1SG of $G$. As $(\gamma, \eta)$ was taken to be an arbitrary element, theorem holds for all $(\gamma, \eta) \in A \times B$. G.

Thus, $\mathscr{G}_{1}^{*} \wedge \mathscr{G}_{2}^{*}=\mathscr{G}^{*}=\left\langle I^{*}, J^{*}, A_{1} \times A_{2}\right\rangle$ is a T2SG of

Definition 29. Let $\mathscr{G}_{1}^{*}=\left\langle S_{1}^{*}, T_{1}^{*}, A_{1}\right\rangle$ and $\mathscr{G}_{2}^{*}=\left\langle S_{2}^{*}, T_{2}^{*}, A_{2}\right\rangle$ be two T2SG of $G$. The OR operation of $\mathscr{G}_{1}^{*}$ and $\mathscr{G}_{2}^{*}$ is denoted by $\mathscr{G}_{1}^{*} \vee \mathscr{G}_{2}^{*}=\mathscr{G}^{*}=\left\langle I^{*}, J^{*}, A \times B\right\rangle$ and defined by $I^{*}(\gamma, \eta)=$ $S_{1}^{*}(\gamma) \widetilde{V} S_{2}^{*}(\eta)$ and $J^{*}(\gamma, \eta)=T_{1}^{*}(\gamma) \widetilde{\nabla} T_{2}^{*}(\eta)$ for all $(\gamma, \eta) \in$ $A \times B$, where $S_{1}^{*}(\gamma) \widetilde{V} S_{2}^{*}(\eta)$ for all $(\gamma, \eta) \in A \times B$ refers to the usual type-1 soft $O R$ operation between the respective T1SS corresponding to $S_{1}^{*}(\gamma)$ and $S_{2}^{*}(\eta)$, respectively, and $T_{1}^{*}(\gamma) \widetilde{V} T_{2}^{*}(\eta)$ for all $(\gamma, \eta) \in A \times B$ refers to the usual type-1 soft $O R$ operation between the respective T1SS corresponding to $T_{1}^{*}(\gamma)$ and $T_{2}^{*}(\eta)$, respectively.

Example 30. Consider graph $(X, \mathscr{E})$ as shown in Figure 10. 


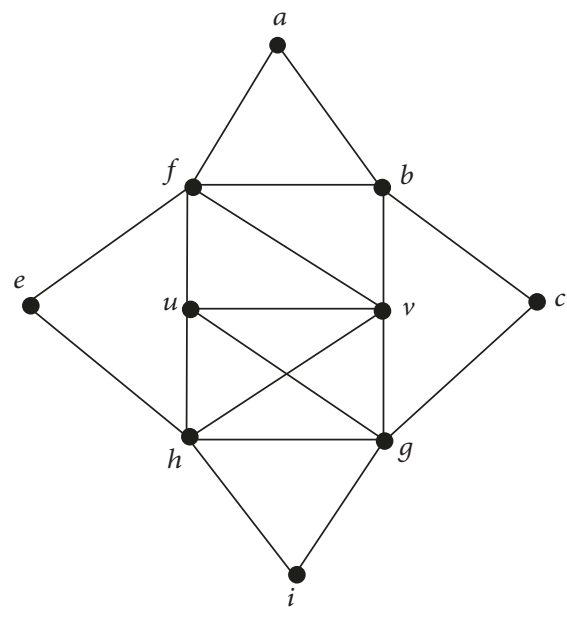

Figure 10: Simple graph.

Let $A=\{e, c\}$ and $B=\{a\}$. Then $N B_{a}=\{f, b\}, N B_{e}=$ $\{f, h\}$, and $N B_{c}=\{g, b\}$. Let $\left[S^{*}, A\right]$ and $\left[T^{*}, A\right]$ be two T2SS, respectively, over $X$ and $\mathscr{E}$ such that $S^{*}(x)=\left(S_{x}, N B_{x}\right)$ and $T^{*}(x)=\left(T_{x}, N B_{x}\right)$ for all $x \in A$, where $S_{x}(z)=\{y \in X \mid$ $z R y \Leftrightarrow d(z, y) \leq 1\}$ and $T_{x}(z)=\left\{\alpha \beta \in \mathscr{E} \mid\{\alpha, \beta\} \subseteq S_{x}(z)\right\}$ for all $z \in N B_{x} \subseteq X$. Now, T2SS $\left[S^{*}, A\right]$ and $\left[T^{*}, A\right]$ are as in the following:

$$
\begin{aligned}
& S^{*}(e)=\left\{\frac{f}{\{a, b, f, u, v, e\}}, \frac{h}{\{e, i, g, h, v, u\}}\right\}, \\
& S^{*}(c)=\left\{\frac{b}{\{b, a, f, c, v\}}, \frac{g}{\{g, h, v, u, c, i\}}\right\}, \\
& T^{*}(e)=\left\{\frac{f}{\{a b, b f, f v, f u, f e, f a, b v, u v\}},\right. \\
& \left.\frac{h}{\{e h, i h, h v, h u, h g, u g, g i, v u, g v\}}\right\}, \\
& T^{*}(c)=\left\{\frac{b}{\{a b, f a, f b, b c, b v, v f\}},\right. \\
& \left.\frac{g}{\{g i, g c, g v, g u, g h, h u, u v, v h, h i\}}\right\} \text {. }
\end{aligned}
$$

Then $\mathscr{G}^{*}=\left\langle S^{*}, T^{*}, A\right\rangle$ is T2SG of $G$.

Let $\left[S^{*}, B\right]$ and $\left[T^{\prime *}, B\right]$ be two T2SS, respectively over $X$ and $\mathscr{E}$ such that $S^{\prime *}(x)=\left(S_{x}^{\prime}, N B_{x}\right)$ and $T^{* *}(x)=\left(T_{x}^{\prime}, N B_{x}\right)$ for all $x \in B$, where $S_{x}^{\prime}(z)=\{y \in X \mid z \mathscr{R} y \Leftrightarrow d(z, y)=2\}$ and $T_{x}^{\prime}(z)=\left\{\alpha \beta \in \mathscr{E} \mid\{\alpha, \beta\} \subseteq S_{x}^{\prime}(z)\right\}$ for all $z \in N B_{x} \subseteq X$. Then T2SS $\left[S^{\prime *}, B\right]$ and $\left[T^{\prime *}, B\right]$ are as in the following:

$$
\begin{aligned}
S^{\prime *}(a) & =\left\{\frac{f}{\{h, g, c\}}, \frac{b}{\{e, h, g, u\}}\right\}, \\
T^{\prime *}(a) & =\left\{\frac{f}{\{g h, g c\}}, \frac{b}{\{g h, h u, h e\}}\right\} .
\end{aligned}
$$

Then $\mathscr{G}^{\prime *}=\left\langle S^{\prime *}, T^{\prime *}, B\right\rangle$ is T2SG of $G$.
The OR operation of $\mathscr{G}^{*}$ and $\mathscr{G}^{\prime *}$ is $\mathscr{G}^{*} \vee \mathscr{G}^{\prime *}=\left\langle I^{*}, J^{*}\right.$, $A \times B\rangle$, where $A \times B=\{(e, a),(c, a)\}$ and

$$
\begin{gathered}
I^{*}(e, a)=\left\{\frac{(f, f)}{\{a, b, f, u, v, e, g, h, c\}}, \frac{(f, b)}{\{a, b, u, v, e, f, h, g\}},\right. \\
\left.\frac{(h, f)}{\{e, i, g, h, v, u, c\}}, \frac{(h, b)}{\{e, i, g, h, v, u\}}\right\} \\
I^{*}(c, a)=\left\{\frac{(b, f)}{\{b, a, v, c, f, h, g\}}, \frac{(b, b)}{\{b, a, v, c, f, e, h, g, u\}},\right. \\
\left.\frac{(g, f)}{\{g, h, v, u, c, i\}}, \frac{(g, b)}{\{g, h, v, c, u, i, e\}}\right\}
\end{gathered}
$$

$J^{*}(e, a)$

$$
=\left\{\frac{(f, f)}{\{a b, b f, a f, f e, e h, h g, g c, b c, b v, f v, v u, f u, u h, u g, v g, v h\}},\right.
$$

$$
\frac{(f, b)}{\{a b, b f, a f, f e, e h, h g, b v, f v, v u, f u, u h, u g, v g, v h\}},
$$

$$
(h, f)
$$

$\overline{\{e h, h g, g c, v u, u h, u g, v g, v h, g i, i h\}}$

$$
\left.\frac{(h, b)}{\{e h, h g, v u, u h, u g, v g, v h, g i, i h\}}\right\}
$$

$J^{*}(c, a)=\left\{\frac{(b, f)}{\{a b, b f, a f, h g, g c, b c, b v, f v, v g, v h\}}\right.$,

$$
\begin{aligned}
& \frac{(b, b)}{\{a b, b f, a f, f e, e h, h g, g c, b c, b v, f v, v u, f u, u h, u g, v g, v h\}}, \\
& \frac{(g, f)}{\{g h, h i, g i, g c, g v, g u, h u, h v, u v\}}, \\
& \left.\frac{(g, b)}{\{g h, h i, g i, g c, g v, g u, h u, h v, u v, e h\}}\right\}
\end{aligned}
$$

The OR operation of $\mathscr{G}^{*}$ and $\mathscr{G}^{\prime *}$ is shown in Figure 11.

Theorem 31. Let $\mathscr{G}_{1}^{*}=\left\langle S^{*}, T^{*}, A_{1}\right\rangle$ and $\mathscr{G}_{2}^{*}=\left\langle S^{\prime *}, T^{\prime *}, A_{2}\right\rangle$ be two T2SG of G. Let T1SS corresponding to $S^{*}(\gamma)$ and $S^{\prime *}(\eta)$ are $\left(S_{\gamma}, N B_{\gamma}\right)$ and $\left(S_{\eta}^{\prime}, N B_{\eta}\right)$ for all $(\gamma, \eta) \in A_{1} \times A_{2}$, respectively. For each $(\gamma, \eta) \in A_{1} \times A_{2}, S_{\gamma}(x) \cap S_{\eta}^{\prime}(y) \neq$ $\emptyset \forall(x, y) \in N B_{\gamma} \times N B_{\eta}$. Then $\mathscr{G}_{1}^{*} \bigvee \mathscr{G}_{2}^{*}$ is a T2SG of $G$.

\section{Proof. Omitted}

Definition 32. Let $\mathscr{G}_{1}^{*}=\left\langle S_{1}^{*}, T_{1}^{*}, A\right\rangle$ and $\mathscr{G}_{2}^{*}=\left\langle S_{2}^{*}, T_{2}^{*}, B\right\rangle$ be two T2SG over $G_{1}$ and $G_{2}$, respectively, such that $A \cap B=\emptyset$ and $N B_{\gamma} \cap N B_{\eta}=\emptyset \forall \gamma \in A, \eta \in B$. The Cartesian product of $\mathscr{G}^{*}$ and $\mathscr{G}^{\prime *}$ is denoted by $\mathscr{G}_{1}^{*} \otimes \mathscr{E}_{2}^{*}$ and defined as $\mathscr{G}_{1}^{*} \otimes$ $\mathscr{G}_{2}^{*}=\left\langle K^{*}, A \times B\right\rangle$, where $K^{*}(\gamma, \eta)=H_{1}^{*}(\gamma) \rtimes H_{2}^{*}(\eta)$ for all $(\gamma, \eta) \in A \times B$ refers to the usual type-1 soft graph Cartesian product between the respective TiSS corresponding to $H_{1}^{*}(\gamma)$ and $H_{1}^{*}(\eta)$, respectively.

Example 33. Consider two graphs $\left(X_{1}, \mathscr{E}_{1}\right)$ and $\left(X_{2}, \mathscr{E}_{2}\right)$ as shown in Figures 12 and 13, respectively. Let $A=\left\{a_{3}, a_{8}\right\}$ and $B=\left\{b_{1}\right\}$. Then $N B_{a_{3}}=\left\{a_{2}, a_{4}\right\}, N B_{a_{8}}=\left\{a_{1}, a_{5}\right\}$, and $N B_{b_{1}}=\left\{b_{2}, b_{6}\right\}$. Let $\left[S^{*}, A\right]$ and $\left[T^{*}, A\right]$ be two T2SS, 

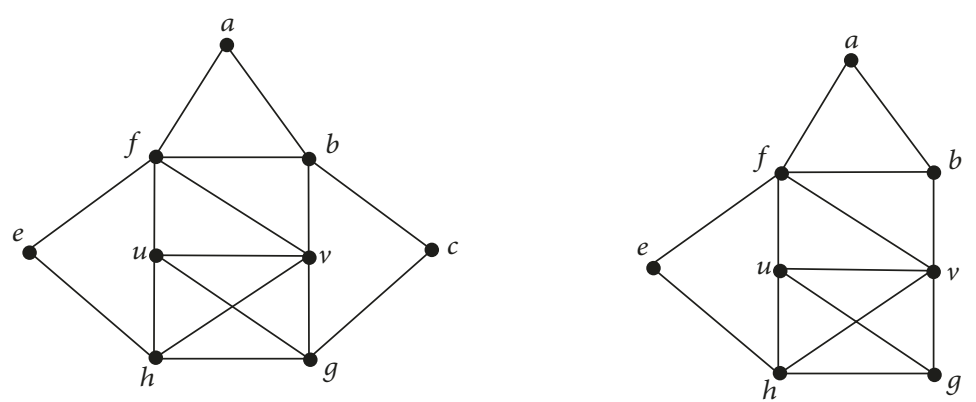

$H_{(e, a)}(f, f)=\left(I_{(e, a)}(f, f), J_{(e, a)}(f, f)\right)$

$H_{(e, a)}(f, b)=\left(I_{(e, a)}(f, b), J_{(e, a)}(f, b)\right)$
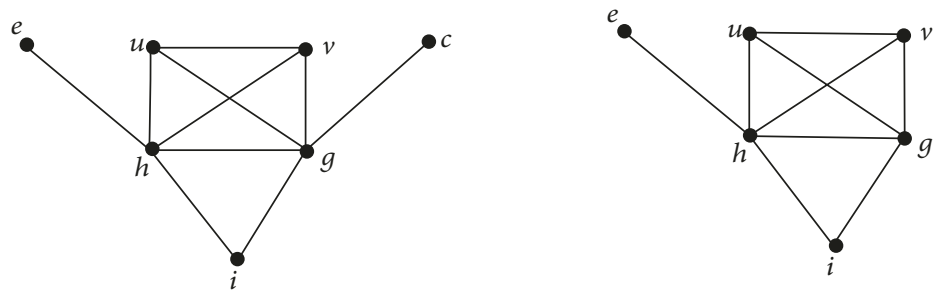

$\left.\frac{H_{(e, a)}(h, f)=\left(I_{(e, a)}(h, f), J_{(e, a)}(h, f)\right) \quad H_{(e, a)}(h, b)=\left(I_{(e, a)}(h, b), J_{(e, a)}\right.}{}(h, b)\right)$
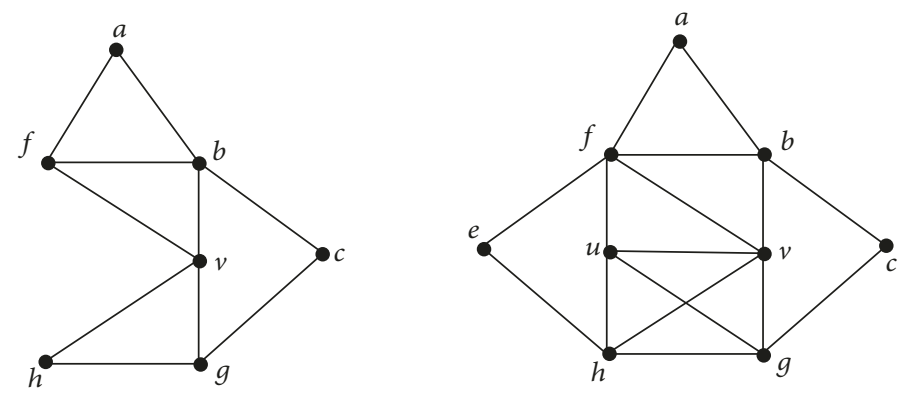

$H_{(c, a)}(b, f)=\left(I_{(c, a)}(b, f), J_{(c, a)}(b, f)\right)$

$H_{(c, a)}(b, b)=\left(I_{(c, a)}(b, b), J_{(c, a)}(b, b)\right)$
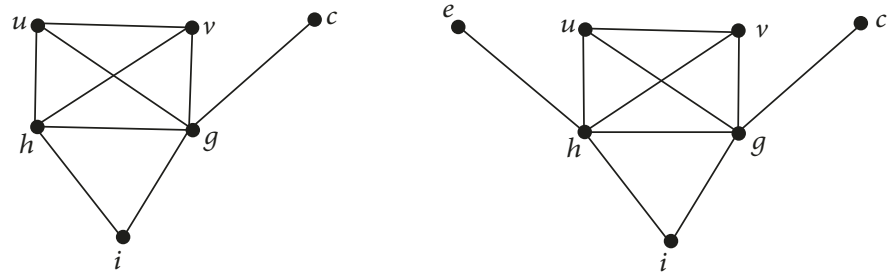

$H_{(c, a)}(g, f)=\left(I_{(c, a)}(g, f), J_{(c, a)}(g, f)\right)$

$$
H_{(c, a)}(g, b)=\left(I_{(c, a)}(g, b), J_{(c, a)}(g, b)\right)
$$

$H^{*}(c, a)=\left(I^{*}(c, a), J^{*}(c, a)\right)$

FIGURE 11: OR operations on $\mathscr{G}^{*}$ and $\mathscr{G}^{* \prime}$.

respectively, over $X_{1}$ and $\mathscr{E}_{1}$ such that $S^{*}(x)=\left(S_{x}, N B_{x}\right)$ and $T^{*}(x)=\left(T_{x}, N B_{x}\right)$ for all $x \in A$, where $S_{x}(z)=\left\{y \in X_{1} \mid\right.$ $\left.z \mathscr{R} y \Leftrightarrow d(z, y)=\operatorname{rad}\left(G_{1}\right)\right\}$ and $T_{x}(z)=\left\{\alpha \beta \in \mathscr{E}_{1} \mid\{\alpha, \beta\} \subseteq\right.$ $\left.S_{x}(z)\right\}$ for all $z \in N B_{x} \subseteq X_{1}$. Now, T2SS $\left[S^{*}, A\right]$ and $\left[T^{*}, A\right]$ are as in the following:

$$
\begin{aligned}
& S^{*}\left(a_{8}\right)=\left\{\frac{a_{1}}{\left\{a_{4}, a_{5}, a_{3}\right\}}, \frac{a_{5}}{\left\{a_{1}, a_{2}, a_{3}\right\}}\right\}, \\
& S^{*}\left(a_{3}\right)=\left\{\frac{a_{2}}{\left\{a_{4}, a_{5}, a_{8}, a_{7}\right\}}, \frac{a_{4}}{\left\{a_{1}, a_{2}, a_{8}\right\}}\right\},
\end{aligned}
$$




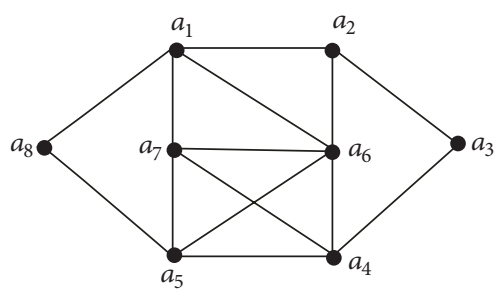

FIGURE 12: Simple graph $\left(X_{1}, \mathscr{E}_{1}\right)$.

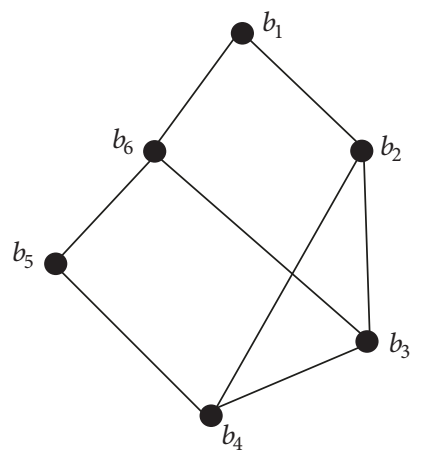

FIGURE 13: Simple graph $\left(X_{2}, \mathscr{E}_{2}\right)$.

$$
\begin{aligned}
& T^{*}\left(a_{8}\right)=\left\{\frac{a_{1}}{\left\{a_{4} a_{5}, a_{4} a_{3}\right\}}, \frac{a_{5}}{\left\{a_{1} a_{2}, a_{2} a_{3}\right\}}\right\}, \\
& T^{*}\left(a_{3}\right)=\left\{\frac{a_{2}}{\left\{a_{4} a_{5}, a_{5} a_{8}, a_{5} a_{7}\right\}}, \frac{a_{4}}{\left\{a_{1} a_{2}, a_{1} a_{8}\right\}}\right\} .
\end{aligned}
$$

Then $\mathscr{G}^{*}=\left\langle S^{*}, T^{*}, A\right\rangle$ is T2SG of $G_{1}$.

Let $\left[S^{\prime *}, B\right]$ and $\left[T^{\prime *}, B\right]$ be two T2SS, respectively, over $X_{2}$ and $\mathscr{E}_{2}$ such that $S^{\prime *}(x)=\left(S_{x}^{\prime}, N B_{x}\right)$ and $T^{\prime *}(x)=\left(T_{x}^{\prime}, N B_{x}\right)$ for all $x \in B$, where $S_{x}^{\prime}(z)=\left\{y \in X_{2} \mid z \mathscr{R} y \Leftrightarrow d(z, y)>1\right\}$ and $T_{x}^{\prime}(z)=\left\{\alpha \beta \in \mathscr{E}_{2} \mid\{\alpha, \beta\} \subseteq S_{x}^{\prime}(z)\right\}$ for all $z \in$ $N B_{x} \subseteq X_{2}$. Then T2SS $\left[S^{*}, B\right]$ and $\left[T^{\prime *}, B\right]$ are as in the following:

$$
\begin{aligned}
& S^{*}\left(b_{1}\right)=\left\{\frac{b_{6}}{\left\{b_{2}, b_{4}\right\}}, \frac{b_{2}}{\left\{b_{5}, b_{6}\right\}}\right\}, \\
& T^{* *}\left(b_{1}\right)=\left\{\frac{b_{6}}{\left\{b_{2} b_{4}\right\}}, \frac{b_{2}}{\left\{b_{6} b_{5}\right\}}\right\} .
\end{aligned}
$$

Then $\mathscr{G}^{\prime *}=\left\langle S^{\prime *}, T^{* *}, B\right\rangle$ is T2SG of $G_{2}$.

The Cartesian product of $\mathscr{G}^{*}$ and $\mathscr{G}^{\prime *}$ is $\mathscr{G}^{*} \rtimes \mathscr{G}^{\prime *}=\left\langle K^{*}\right.$, $A \times B\rangle$, where $A \times B=\left\{\left(a_{3}, b_{1}\right)\left(a_{8}, b_{1}\right)\right\}$ and

$$
\begin{aligned}
& I^{*}\left(a_{8}, b_{1}\right)=\left\{\frac{\left(a_{1}, b_{6}\right)}{\left\{\left(a_{4}, b_{2}\right),\left(a_{5}, b_{2}\right),\left(a_{3}, b_{2}\right),\left(a_{4}, b_{4}\right),\left(a_{5}, b_{4}\right),\left(a_{3}, b_{4}\right)\right\}}, \frac{\left(a_{5}, b_{6}\right)}{\left\{\left(a_{1}, b_{2}\right),\left(a_{2}, b_{2}\right),\left(a_{3}, b_{2}\right),\left(a_{1}, b_{4}\right),\left(a_{2}, b_{4}\right),\left(a_{3}, b_{4}\right)\right\}}, \frac{\left(a_{5}, b_{2}\right)}{\left\{\left(a_{1}, b_{5}\right),\left(a_{2}, b_{5}\right),\left(a_{3}, b_{5}\right),\left(a_{1}, b_{6}\right),\left(a_{2}, b_{6}\right),\left(a_{3}, b_{6}\right)\right\}},\right. \\
& \left.\quad \frac{\left(a_{1}, b_{2}\right)}{\left\{\left(a_{4}, b_{5}\right),\left(a_{5}, b_{5}\right),\left(a_{3}, b_{5}\right),\left(a_{4}, b_{6}\right),\left(a_{5}, b_{6}\right),\left(a_{3}, b_{6}\right)\right\}}\right\} \\
& J^{*}\left(a_{8}, b_{1}\right) \\
& \quad=\left\{\frac{\left(a_{1}, b_{6}\right)}{\left\{\left(\left(a_{4}, b_{2}\right),\left(a_{5}, b_{2}\right)\right),\left(\left(a_{4}, b_{2}\right),\left(a_{3}, b_{2}\right)\right),\left(\left(a_{5}, b_{2}\right),\left(a_{3}, b_{2}\right)\right),\left(\left(a_{4}, b_{4}\right),\left(a_{5}, b_{4}\right)\right),\left(\left(a_{4}, b_{4}\right),\left(a_{3}, b_{4}\right)\right),\left(\left(a_{5}, b_{4}\right),\left(a_{3}, b_{4}\right)\right),\left(\left(a_{4}, b_{2}\right),\left(a_{4}, b_{4}\right)\right),\left(\left(a_{5}, b_{2}\right),\left(a_{5}, b_{4}\right)\right),\left(\left(a_{3}, b_{2}\right),\left(a_{3}, b_{4}\right)\right)\right\}},\right. \\
& \frac{\left.\left(\left(a_{1}, b_{2}\right),\left(a_{2}, b_{2}\right)\right),\left(\left(a_{1}, b_{2}\right),\left(a_{3}, b_{2}\right)\right),\left(\left(a_{2}, b_{2}\right),\left(a_{3}, b_{2}\right)\right),\left(\left(a_{1}, b_{4}\right),\left(a_{2}, b_{4}\right)\right),\left(\left(a_{1}, b_{4}\right),\left(a_{3}, b_{4}\right)\right),\left(\left(a_{2}, b_{4}\right),\left(a_{3}, b_{4}\right)\right),\left(\left(a_{1}, b_{2}\right),\left(a_{1}, b_{4}\right)\right),\left(\left(a_{2}, b_{2}\right),\left(a_{2}, b_{4}\right)\right),\left(\left(a_{3}, b_{2}\right),\left(a_{3}, b_{4}\right)\right)\right\}}{}, \\
& \frac{\left(\left(\left(a_{1}, b_{5}\right),\left(a_{2}, b_{5}\right)\right),\left(\left(a_{1}, b_{5}\right),\left(a_{3}, b_{5}\right)\right),\left(\left(a_{2}, b_{5}\right),\left(a_{3}, b_{5}\right)\right),\left(\left(a_{1}, b_{6}\right),\left(a_{2}, b_{6}\right)\right),\left(\left(a_{1}, b_{6}\right),\left(a_{3}, b_{6}\right)\right),\left(\left(a_{2}, b_{6}\right),\left(a_{3}, b_{6}\right)\right),\left(\left(a_{1}, b_{5}\right),\left(a_{1}, b_{6}\right)\right),\left(\left(a_{2}, b_{5}\right),\left(a_{2}, b_{6}\right)\right),\left(\left(a_{3}, b_{5}\right),\left(a_{3}, b_{6}\right)\right)\right\}}{\left.\left(\left(a_{4}, b_{5}\right),\left(a_{5}, b_{5}\right)\right),\left(\left(a_{4}, b_{5}\right),\left(a_{3}, b_{5}\right)\right),\left(\left(a_{5}, b_{5}\right),\left(a_{3}, b_{5}\right)\right),\left(\left(a_{4}, b_{6}\right),\left(a_{5}, b_{6}\right)\right),\left(\left(a_{4}, b_{6}\right),\left(a_{3}, b_{6}\right)\right),\left(\left(a_{5}, b_{6}\right),\left(a_{3}, b_{6}\right)\right),\left(\left(a_{4}, b_{5}\right),\left(a_{4}, b_{6}\right)\right),\left(\left(a_{5}, b_{5}\right),\left(a_{5}, b_{6}\right)\right),\left(\left(a_{3}, b_{5}\right),\left(a_{3}, b_{6}\right)\right)\right\}},
\end{aligned}
$$

$H^{*}\left(a_{8}, b_{1}\right)$ is shown in Figure 14. Similarly, we can compute $H^{*}\left(a_{3}, b_{1}\right)$ and show the respective graphs.

Theorem 34. Let $\mathbb{Q}$ be the Cartesian product of two simple graphs $G_{1}$ and $G_{2}$. Let $\mathscr{G}_{1}^{*}$ and $\mathscr{G}_{2}^{*}$ be two T2SG of $G_{1}$ and $G_{2}$, respectively. Then $\mathscr{G}_{1}^{*} \otimes \mathscr{G}_{2}^{*}=\left\langle K^{*}, A \times B\right\rangle$ is a T2SG of $Q$.

Proof. The Cartesian product of two T2SG $\mathscr{G}_{1}^{*}$ and $\mathscr{G}_{2}^{*}$ is $\mathscr{G}_{1}^{*} \otimes$ $\mathscr{G}_{2}^{*}=\left\langle K^{*}, A \times B\right\rangle$, where $K^{*}(\gamma, \eta)=H_{1}^{*}(\gamma) \rtimes H_{2}^{*}(\eta)$ for all $(\gamma, \eta) \in A \times B$ refers to the usual type-1 soft graphs Cartesian product between the respective T1SS corresponding to $H_{1}^{*}(\gamma)$ and $H_{1}^{*}(\eta)$, respectively.

Given that $\mathscr{G}_{1}^{*}$ is a T2SG of $G_{1}$, then T1SG corresponding to $H_{1}^{*}(\gamma) \forall \gamma \in A$ is a connected T1SG of $G_{1}$. Also, given that $\mathscr{G}_{2}^{*}$ is a T2SG of $G_{2}$, then T1SG corresponding to $H_{2}^{*}(\eta) \forall \eta \in B$ is a connected T1SG of $G_{2}$. This implies that T1SG corresponding to $K^{*}(\gamma, \eta) \forall(\gamma, \eta) \in A \times B$ is a Cartesian product of two connected T1SG. Therefore, T1SG corresponding to $K^{*}(\gamma, \eta)$ is connected T1SG of Q , for all $(\gamma, \eta) \in A \times B$. Thus, $\mathscr{G}_{1}^{*} \otimes \mathscr{G}_{2}^{*}=\left\langle K^{*}, A \times B\right\rangle$ is a T2SG of $\mathbb{Q}$.

\section{An Application of Type-2 Soft Graphs}

It is well known that graph theory has many applications in computer networks and communication networks. In this manner, our approach is noteworthy to enhance the study of networks. In this section, we contemplated an application of T2SG in communication networks.

It is necessary to explain some new notions of T2SS that will be used in this section. Let $\left[S^{*}, A\right]$ and $\left[S^{*}, B\right]$ be two T2SS over $U$. Let, for each $\left(\gamma_{k}, \eta_{k^{\prime}}\right) \in A \times B,\left(S_{\gamma_{k}}, N B_{\gamma_{k}}\right)$ and 

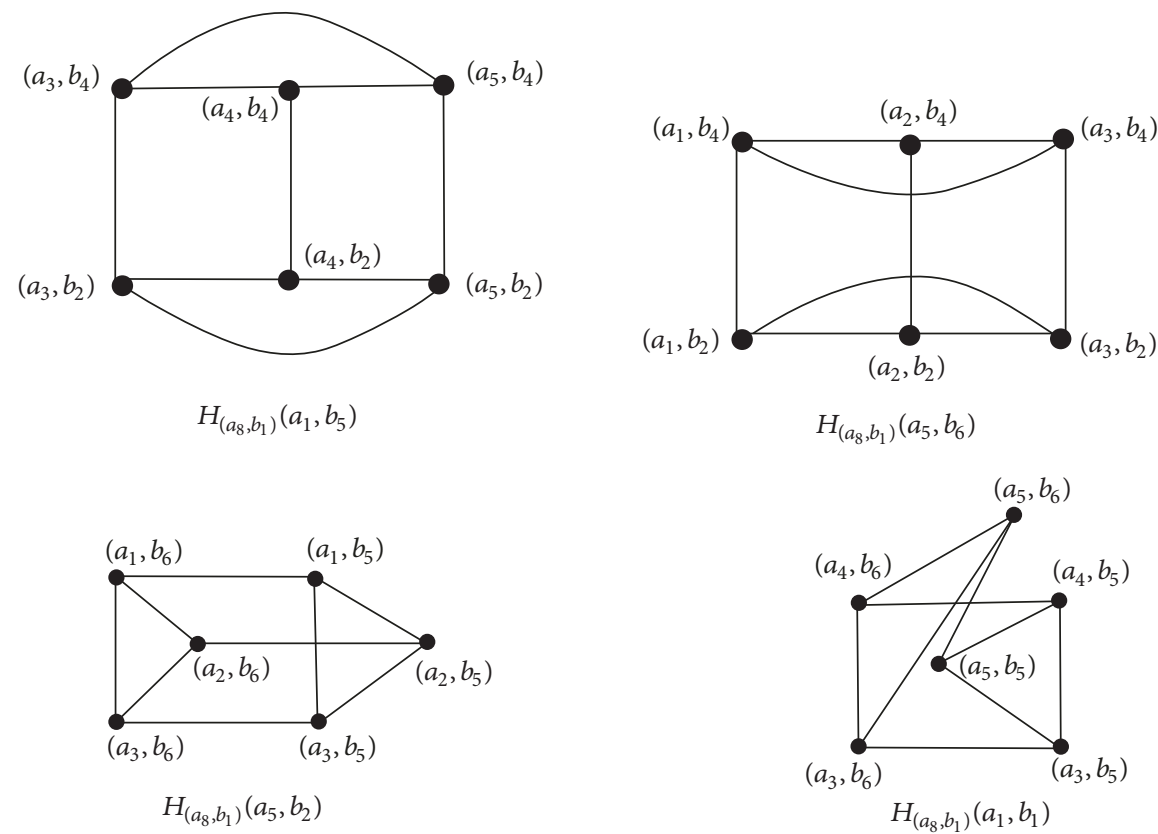

FiguRE 14: $H^{*}\left(a_{8}, b_{1}\right)$.

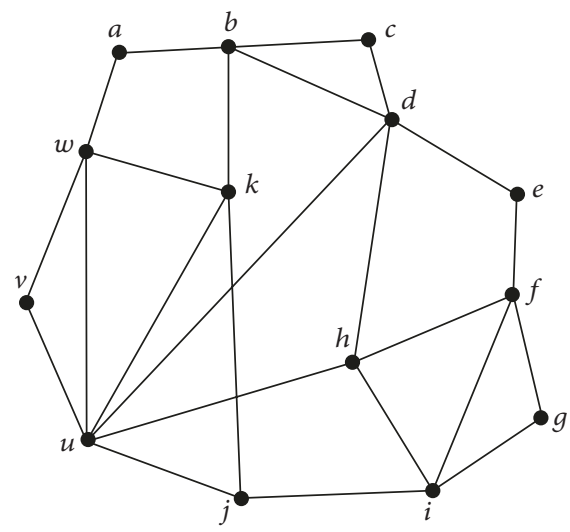

FIGURE 15: Communication network.

$\left(S_{\eta_{k^{\prime}}}^{\prime}, N B_{\eta_{k^{\prime}}}\right)$ be the T1SS corresponding to $S^{*}\left(\gamma_{k}\right)$ and $S^{\prime *}\left(\eta_{k^{\prime}}\right)$, respectively. A correlation table of T2SS is an $m \times n$ table in which the entry $s_{k_{t} k_{j}^{\prime}}$ is a resultant of $S_{\gamma_{k}}\left(x_{t}\right) \cap S_{\eta_{k^{\prime}}}^{\prime}\left(x_{j}\right)$ for all $\left(x_{t}, x_{j}\right) \in N B_{\gamma_{k}} \times N B_{\eta_{k^{\prime}}}\left(k=1,2, \ldots, m^{\prime}\right.$ and $\left.k^{\prime}=1,2, \ldots, n^{\prime}\right)$. There are $m^{\prime} \cdot n^{\prime}=q$, partitions in correlation table, where each partition represents an AND operation on respective T1SS corresponding to $S^{*}\left(\gamma_{k}\right)$ and $S^{\prime *}\left(\eta_{k^{\prime}}\right)$ for all $\left(\gamma_{k}, \eta_{k^{\prime}}\right) \in$ $A \times B$. The R-union in a partition is the row-wise union of entries and the $C$-intersection in a partition is the columnwise intersection of entries.

Consider a communication network of 14 communications towers (see Figure 15), where each tower is represented as a vertex and each communication link between towers is represented with an edge in a resulting graph. The communication among the towers in the network is done by using two types of messages: Hello (used for sending information to neighbors) and Data (used for data transmission). There are two types of towers in the network: (i) working as a base station and working in subnetworks and (ii) working in subnetworks. Hello, messages are only considered by one-hop neighbors, while the Data messages are representing sending and gathering information from subnetworks and therefore must be transmitted in a multihop manner towards the base stations and towards the subnetworks.

Two communications companies are using this network of towers, and each one uses a set of base stations for communication: the first company is using towers $a, e$ as base stations, while the second company is using towers $c, g$ as base stations. Let $A=\{a, e\}$ and $B=\{c, g\}$. For any base station $\ell$ messaging is as follows:

(i) $\ell$ sends Hello messages and their neighbors $N B_{\ell}$ send these messages as Data messages towards the set of all towers in the network.

(ii) Also, the neighbors of $\ell$ gather messages from the whole network and then send these messages as Hello messages to $\ell$.

To analyze the weak service (messaging), both companies make a survey on their sets of base stations. The region (towers and the links between towers) of weak service of $A$ and $B$ may be defined in the form of T2SG.

The region of weak service for $A$ is as follows: for base station $a \in A$, region of weak messaging is defined as a T1SS, $S_{a}(z)=\{y \in X \mid y \mathscr{R} z \Leftrightarrow d(y, z) \leq 2\}$ and $T_{a}(z)=\{u v \in \mathscr{E} \mid$ $\left.\{u, v\} \subseteq S_{a}(z)\right\} \forall z \in N B_{a}$.

For base station $e \in A$, region of weak messaging is defined as a T1SS, $S_{e}\left(z^{\prime}\right)=\left\{y^{\prime} \in X \mid y^{\prime} \mathscr{R} z^{\prime} \Leftrightarrow d\left(y^{\prime}, z^{\prime}\right)=1\right\}$ 
TABLE 2: $\left(A \wedge^{X} B\right)$ correlation table.

\begin{tabular}{|c|c|c|c|c|c|}
\hline \multirow{2}{*}{$\mathscr{B}$} & \multirow{2}{*}{$\mathscr{A}$} & \multicolumn{2}{|c|}{$S^{*}(a)$} & \multicolumn{2}{|c|}{$S^{*}(e)$} \\
\hline & & $S_{a}(w)$ & $S_{a}(b)$ & $S_{e}(d)$ & $S_{e}(f)$ \\
\hline \multirow{2}{*}{$S^{\prime *}(c)$} & $S_{c}^{\prime}(b)$ & $\{a, w, u, d, h, k, j\}$ & $\{a, c, d, k, u, w, j, h, e\}$ & $\{c, h, e, u\}$ & $\{e, h\}$ \\
\hline & $S_{c}^{\prime}(d)$ & $\{a, b, k, u, j, h, v, w\}$ & $\{a, b, c, k, u, w, j, h, e\}$ & $\{b, c, h, e, u\}$ & $\{e, i, h\}$ \\
\hline \multirow{2}{*}{$S^{\prime *}(g)$} & $S_{g}^{\prime}(i)$ & $\{d, u, k, h, j\}$ & $\{e, d, u, k, h, j\}$ & $\{e, u, h\}$ & $\{e, h, g\}$ \\
\hline & $S_{g}^{\prime}(f)$ & $\{u, d, j, h\}$ & $\{e, u, d, j, h\}$ & $\{u, h, e\}$ & $\{e, i, h, g\}$ \\
\hline
\end{tabular}

and $T_{e}\left(z^{\prime}\right)=\left\{u^{\prime} v^{\prime} \in \mathscr{E} \mid\left\{u^{\prime}, v^{\prime}\right\} \subseteq S_{e}\left(z^{\prime}\right)\right\} \forall z^{\prime} \in N B_{e}$. Then T2SS are as follows:

$$
\begin{aligned}
& S^{*}(a)=\left\{\frac{w}{\{a, b, d, h, k, u, j, v, w\}}, \frac{b}{\{a, b, c, d, k, u, w, j, h, e\}}\right\}, \\
& S^{*}(e)=\left\{\frac{d}{\{b, c, h, e, u\}}, \frac{f}{\{e, i, h, g\}}\right\}, \\
& T^{*}(a) \\
& =\left\{\frac{w}{\{a b, b d, a w, b k, w k, w u, w v, u v, u k, u d, u j, u h, k j, h d\}},\right. \\
& \left.\frac{b}{\{a b, b c, b d, c d, d h, a w, b k, k u, u h, d e, u d, u w, u j, w k, k j\}}\right\}, \\
& S^{* *}(c)=\left\{\frac{b}{\{a, w, c, u, k, d, e, h, j\}}, \frac{d}{\{a, b, c, w, v, u, j, i, k, f, h, e\}}\right\}, \\
& S^{\prime *}(g)=\left\{\frac{i}{\{g, f, e, h, d, j, u, k\}}, \frac{f}{\{e, d, h, u, g, i, j\}}\right\}, \\
& T^{\prime *}(c)=\left\{\frac{b}{\{a w, w k, w u, u k, u d, u j, u h, k j, d c, d e, h d\}}, \frac{d}{\{f i, f e, a b, b c, b k, a w, u v, u h, u j, i j, f h, k w, v w, h i, j k, u k, u w\}}\right\}, \\
& T^{*}(g)=\left\{\frac{i}{\{e d, d u, k u, g f, f e, h d, h f, h u, u j, k j\}}, \frac{f}{\{u j, u d, e d, h d, h i, i g, j i, u h\}}\right\} .
\end{aligned}
$$
of $G$.

One can check that $\mathscr{G}^{\prime *}=\left(G, S^{\prime *}, T^{\prime *}, B, N B_{B}\right)$ is a T2SG

To analyze the region (towers and the links between towers) in which both the sets of base stations are incorporated for very fragile service in the communication network, first, construct correlation tables of $\left(A \wedge^{X} B\right)$ and $\left(A \wedge^{\mathscr{E}} B\right)$ for vertices and edges, respectively. Let $\left(\gamma_{k}, \eta_{k^{\prime}}\right) \in A \times B$. In the correlation Table 2 entry $s_{k_{t} k_{j}^{\prime}}$ is a resultant of $S_{\gamma_{k}}\left(x_{l}\right) \cap S_{\eta_{k^{\prime}}}^{\prime}\left(x_{j}\right)$ and in the correlation Table 3 entry $t_{k_{l} k_{1}^{\prime}}$ is a resultant of $T_{\gamma_{k}}\left(x_{l}\right) \cap T_{\eta_{k^{\prime}}}^{\prime}\left(x_{j}\right)$ for all $\left(x_{l}, x_{j}\right) \in N B_{\gamma_{k}} \times N B_{\eta_{k^{\prime}}}(k=1,2$ and $\left.k^{\prime}=1,2\right)$. There are $2 \cdot 2=4$, partitions in the correlation Table 2, where each partition represents an AND operation on respective T1SS corresponding to $S^{*}\left(\gamma_{k}\right)$ and $S^{\prime *}\left(\eta_{k^{\prime}}\right)$, respectively. Also, there are $2 \cdot 2=4$, partitions in the correlation Table 3, where each partition represents an AND operation on the respective T1SS corresponding to $T^{*}\left(\gamma_{k}\right)$ and $T^{\prime *}\left(\eta_{k^{\prime}}\right)$, respectively.

Take $R$-union of each partition in Table 2 and represent it in Table 4. Similarly, compute Table 5 from Table 3.

Next, take $C$-intersection on each partition in Table 4 and represent it in Table 6. Similarly, compute Table 7 from Table 5 .

Take $R$-union on Table 6 and represent it in $2 \times 1$ matrix of vertices. Sequentially, obtain a $2 \times 1$ matrix of edges from Table 7.

Select the subgraph that is involved in extremely poor communications for both companies integratedly. One can easily check that the vertices $u, h, e$, and $j$ appear maximum times (twice) in $2 \times 1$ matrix of vertices and the edges $u j$ and 
TABLE 3: $\left(A \wedge^{\mathscr{E}} B\right)$ correlation table.

\begin{tabular}{lccccc}
\hline $\mathscr{B}$ & $\mathscr{A}$ & & $T^{*}(a)$ & \multicolumn{2}{c}{$T^{*}(e)$} \\
& & $T_{a}(w)$ & $T_{a}(b)$ & $T_{e}(d)$ & $T_{e}(f)$ \\
\hline \multirow{2}{*}{$T^{* *}(c)$} & $T_{c}^{\prime}(b)$ & $\{a w, w k, w u, u k, u d, u j, u h, k j, h d\}$ & $\{a w, w k, w u, u k, d c, h d, d e, u h, u j, k j, u d\}$ & $\{h u\}$ & $\emptyset$ \\
& $T_{c}^{\prime}(d)$ & $\{a b, a w, b k, w k, w u, w v, u v, u k, u j, u h, k j\}$ & $\{a b, b c, a w, b k, k u, u h, w u, w k, u j, k j\}$ & $\{h u, b c\}$ & $\{h i\}$ \\
\hline \multirow{2}{*}{$T^{\prime *}(g)$} & $T_{g}^{\prime}(i)$ & $\{u d, k u, k j, u h, u j, h d\}$ & $\{d e, u k, u d, h d, j u, u h, k j\}$ & $\{h u\}$ & $\emptyset$ \\
& $T_{g}^{\prime}(f)$ & $\{u j, u d, u h, h d\}$ & $\{u j, u h, h d, d e\}$ & $\{h u\}$ & $\{h i, g i\}$ \\
\hline
\end{tabular}

TABLe 4: R-union on $A \wedge^{X} B$ correlation table.

\begin{tabular}{lc}
\hline$R$-union of $S^{*}(a) \tilde{\wedge} S^{\prime *}(c)$ & $R$-union of $S^{*}(e) \tilde{\wedge} S^{\prime *}(c)$ \\
\hline$a, w, u, d, h, k, j, c, e\}$ & $\{e, h, c, u\}$ \\
$\{a, b, w, k, u, j, h, v, c, e\}$ & $\{e, h, c, u, b, i\}$ \\
\hline$R$-union of $S^{*}(a) \widetilde{\wedge} S^{\prime *}(g)$ & $R$-union of $S^{*}(e) \widetilde{\wedge} S^{\prime *}(g)$ \\
\hline$\{e, d, u, k, j, h\}$ & $\{e, u, h, g\}$ \\
$\{u, d, j, h, e\}$ & $\{u, h, e, i, g\}$ \\
\hline
\end{tabular}

uh appear twice in $2 \times 1$ matrix of edges. Thus, the very fragile communication for both companies integratedly is shown in Figure 16. Eventually, both companies are incorporated to fix the fragile communication on resultant subgraph.

$$
\begin{aligned}
& R \text {-Union (Table 6) }=\left[\begin{array}{c}
\{a, w, u, h, k, j, c, e\} \\
\{u, d, e, j, h, g\}
\end{array}\right], \\
& R \text {-Union (Table 7) }=\left[\begin{array}{c}
\{a w, w k, u w, u k, u j, u h\} \\
\{u d, u j, h d, u h, d e\}
\end{array}\right] .
\end{aligned}
$$

We now present the general procedure of our method which is used in our application from the following algorithm.

\section{Algorithm}

(i) Input the two sets of base stations in a communication network.

(ii) Define T2SG on each set of base stations, according to the weak communication messaging, that is, Hello messages and Data messages.

(iii) Take AND operation on T2SG represents it in the correlation tables:

(1) Table 1 represents AND operation on two T2SS of the vertices and it contains $m^{\prime} \cdot n^{\prime}$ partitions, where each partition is an AND operation of respective TISS corresponding to $S^{*}\left(\gamma_{k}\right)$ and $S^{\prime *}\left(\eta_{k^{\prime}}\right)$, respectively, $\left(k=1,2, \ldots, m^{\prime}, k^{\prime}=\right.$ $\left.1,2, \ldots, n^{\prime}\right)$.

(2) Table 2 represents AND operation on two T2SS of the edges and it contains $m^{\prime} \cdot n^{\prime}$ partitions, where each partition is an AND operation of respective TISS corresponding to $T^{*}\left(\gamma_{k}\right)$ and $T^{\prime *}\left(\eta_{k^{\prime}}\right)$, respectively, $\left(k=1,2, \ldots, m^{\prime}, k^{\prime}=\right.$ $\left.1,2, \ldots, n^{\prime}\right)$.

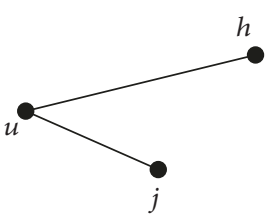

FIGURE 16: Resultant graph.

(iv) Take R-union on each partition of Table 1, representing it in Table 3. Similarly, compute Table 4 from Table 2.

(v) Take C-intersection on each partition in Table 3, representing it in Table 5. Similarly, compute Table 6 from Table 4.

(vi) Take $R$-union on Table 5 represents an $n^{\prime} \times 1$ matrix of vertices. Similarly, obtain an $n^{\prime} \times 1$ matrix of edges from Table 6.

(vii) Find the vertices that appear maximum " $n$ " " times in the $n^{\prime} \times 1$ matrix of vertices, and then find the edges that appear " $n$ "” times in the $n^{\prime} \times 1$ matrix of edges. Note that $n^{*} \leq n^{\prime}$.

(viii) By using vertices and edges that are obtained from step (vii), represent a graph of very unwell communications for both sets of base stations integratedly. It may be a connected or disconnected graph.

Finally, both companies incorporated to fix the fragile communication on resultant subgraph. Similarly, we can present an algorithm for more than two sets of base stations in a network.

\section{Conclusion}

In the above study, we have presented an application of type-2 soft sets in graph theory. We have introduced vertexneighbors based type-2 soft sets over $X$ and $\mathscr{E}$. We have introduced some operations on type-2 soft graphs. We have described a practical application of type-2 soft graphs in communication networks and present procedure as an algorithm. The type- 2 soft graphs are more general and hence a more efficient tool as compared to type-1 soft graphs. The idea presented in this paper has practical applications in the social networks and computer networks. 
TABLE 5: $R$-union on $A \wedge^{\mathscr{E}} B$ correlation table.

\begin{tabular}{lr}
\hline$R$-union of $T^{*}(a) \tilde{\wedge} T^{\prime *}(c)$ & $R-u n i o n$ of $T^{*}(e) \tilde{\wedge} T^{\prime *}(c)$ \\
\hline$\{a w, w k, w u, u k, u d, u j, u h, h d, d c, d e\}$ & $\{h u\}$ \\
$\{a b, a w, b k, w k, w u, w v, u v, u k, u j, u h, k j, b c\}$ & $\{h u, b c, h i\}$ \\
\hline$R-u n i o n$ of $T^{*}(a) \tilde{\wedge} T^{*}(g)$ & $R-u n i o n$ of $T^{*}(e) \tilde{\wedge} T^{* *}(g)$ \\
\hline$\{u k, u d, d e, j k, u h, h d, u j\}$ & $\{h u\}$ \\
$\{u j, u d, u h, h d, d e\}$ & $\{h u, g i, h i\}$ \\
\hline
\end{tabular}

TABLE 6: C-intersection of R-union of $A \wedge^{X} B$ correlation table.

\begin{tabular}{lr}
\hline$C$-intersection of $R$-union of $S^{*}(a) \tilde{\wedge} S^{*}(c)$ & $C$-intersection of $R$-union of $S^{*}(e) \tilde{\wedge} S^{\prime *}(c)$ \\
\hline$\{a, w, u, h, k, j, c, e\}$ & $\{e, h, c, u\}$ \\
\hline$C$-intersection of $R$-union of $S^{*}(a) \tilde{\wedge} S^{*}(g)$ & $C$-intersection of $R$-union of $S^{*}(e) \tilde{\wedge} S^{* *}(g)$ \\
\hline$\{u, d, e, j, h\}$ & $\{u, h, e, g\}$
\end{tabular}

TABLE 7: C-intersection of $R$-union of $A \wedge^{\mathscr{E}} B$ correlation table.

\begin{tabular}{lc}
\hline$C$-intersection of $R$-union of $T^{*}(a) \tilde{\wedge} T^{\prime *}(c)$ & $C$-intersection of $R$-union of $T^{*}(e) \tilde{\wedge} T^{\prime *}(c)$ \\
\hline$\{a w, w k, u w, u k, u j, u h\}$ & $\{h u\}$ \\
\hline$C$-intersection of $R$-union of $T^{*}(a) \tilde{\wedge} T^{\prime *}(g)$ & $C$-intersection of $R$-union of $T^{*}(e) \tilde{\wedge} T^{\prime *}(g)$ \\
\hline$\{u d, u j, h d, u h, d e\}$ & $\{h u\}$ \\
\hline
\end{tabular}

\section{Conflicts of Interest}

The authors declare that they have no conflicts of interest.

\section{Acknowledgments}

This work was supported by the Natural Science Foundation of Guangdong Province (2016A030313552 and 2016A030307037), the Guangdong Provincial Government to Guangdong International Student Scholarship (yuejiao [2014] 187), and Guangzhou Vocational College of Science and Technology (nos. 2016TD03 and 16YJAZH081).

\section{References}

[1] A. Rosenfeld, "Fuzzy graphs," in Fuzzy Sets and Their Applications, L. A. Zadeh, K. S. Fu, and M. Shimura, Eds., pp. 77-95, Academic Press, New York, NY, USA, 1975.

[2] A. Kauffman, "Introduction a la Theorie des Sous-emsembles Flous, Masson et Cie," vol. 1, 1973.

[3] L. A. Zadeh, "Fuzzy sets," Information and Computation, vol. 8, pp. 338-353, 1965.

[4] P. Bhattacharya, "Some remarks on fuzzy graphs," Pattern Recognition Letters, vol. 6, no. 5, pp. 297-302, 1987.

[5] J. N. Mordeson and P. S. Nair, Fuzzy Graphs and Fuzzy Hypergraphs, vol. 46 of Studies in Fuzziness and Soft Computing, Physica-Verlag HD, Heidelberg, Germany, 2nd edition, 2000.

[6] M. Akram, "Bipolar fuzzy graphs with applications," KnowledgeBased Systems, vol. 39, pp. 1-8, 2013.

[7] M. Akram and W. A. Dudek, "Intuitionistic fuzzy hypergraphs with applications," Information Sciences, vol. 218, pp. 182-193, 2013.

[8] M. Akram and R. Akmal, "Intuitionistic fuzzy graph structures," Kragujevac Journal of Mathematics, vol. 41, pp. 219-237, 2017.
[9] M. Akram and W. A. Dudek, "Interval-valued fuzzy graphs," Computers \& Mathematics with Applications, vol. 61, no. 2, pp. 289-299, 2011.

[10] S. Samanta, M. Pal, H. Rashmanlou, and R. A. Borzooei, "Vague graphs and strengths," Journal of Intelligent and Fuzzy Systems, vol. 30, no. 6, pp. 3675-3680, 2016.

[11] S. Samanta and M. Pal, "Fuzzy Planar Graphs," IEEE Transactions on Fuzzy Systems, vol. 23, no. 6, pp. 1936-1942, 2015.

[12] S. Samanta and M. Pal, "Irregular bipolar fuzzy graphs," International Journal of Fuzzy Systems, vol. 2, pp. 91-102, 2012.

[13] D. Molodtsov, "Soft set theory-first results," Computers \& Mathematics with Applications, vol. 37, no. 4-5, pp. 19-31, 1999.

[14] P. K. Maji, R. Biswas, and A. R. Roy, "Soft set theory," Computers \& Mathematics with Applications, vol. 45, no. 4-5, pp. 555-562, 2003.

[15] H. Aktaş and N. Çağman, "Soft sets and soft groups," Information Sciences, vol. 177, no. 13, pp. 2726-2735, 2007.

[16] M. I. Ali, F. Feng, X. Liu, W. K. Min, and M. Shabir, "On some new operations in soft set theory," Computers \& Mathematics with Applications, vol. 57, no. 9, pp. 1547-1553, 2009.

[17] A. Sezgin and A. O. Atagün, "On operations of soft sets," Computers \& Mathematics with Applications, vol. 61, no. 5, pp. 1457-1467, 2011.

[18] P. K. Maji, R. Biswas, and A. R. Roy, "Fuzzy soft sets," Journal of Fuzzy Mathematics, vol. 9, pp. 589-602, 2001.

[19] M. I. Ali, M. Shabir, and F. Feng, "Representation of graphs based on neighborhoods and soft sets," International Journal of Machine Learning and Cybernetics, vol. 8, no. 5, pp. 1525-1535, 2017.

[20] M. Akram and S. Nawaz, "Operation on soft graph," Fuzzy Information and Engineering, vol. 7, pp. 423-449, 2015.

[21] M. Akram and S. Nawaz, "Certian types of soft graphs," Scientific Bulletin-University Politehnica of Bucharest, Series A, vol. 78, pp. 67-82, 2016.

[22] M. Akram and S. Nawaz, "On fuzzy soft graphs," Italian Journal of Pure and Applied Mathematics, no. 34, pp. 497-514, 2015. 
[23] J. A. Bondy and U. S. R. Murty, Graph Theory with Applications, Macmillan Press, New York, NY, USA, 1976.

[24] A. E. Brouwer, A. M. Cohen, and A. Neumaier, Distance-Regular Graphs, vol. 18, Springer, New York, NY, USA, 1989.

[25] W. T. Tutte, Graph Theory as I Have Known It, Oxford University Press, Oxford, England, 1998.

[26] R. Chatterjeea, P. Majumdar, and S. K. Samanta, “Type-2 soft sets,” Journal of Intelligent \& Fuzzy Systems, vol. 29, pp. 885-898, 2015.

[27] R. Chatterjeea, P. Majumdar, and S. K. Samanta, "Distance, entropy and similarity measures of Type-2 soft sets," 2016, https://arxiv.org/abs/1612.06603. 

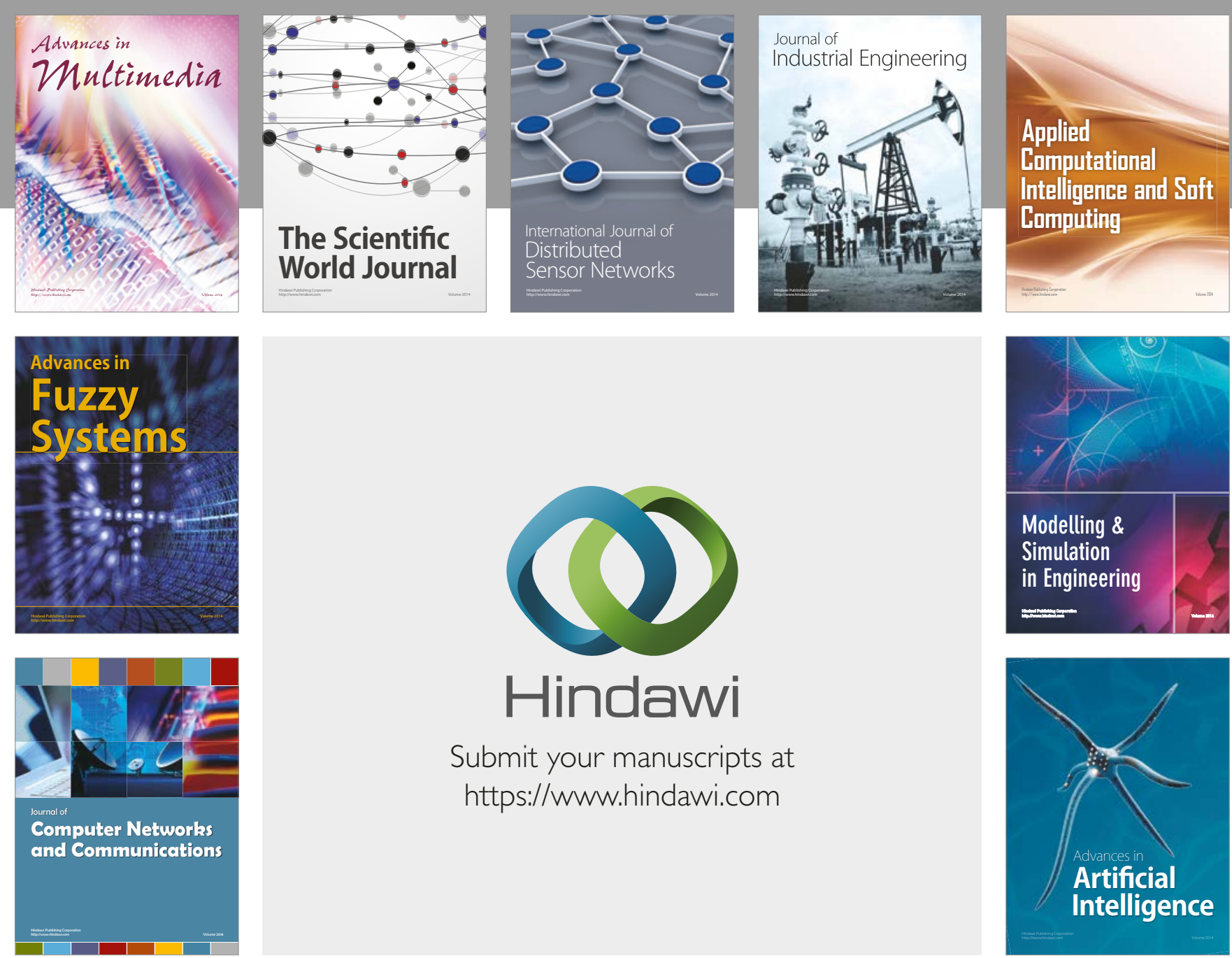

\section{Hindawi}

Submit your manuscripts at

https://www.hindawi.com
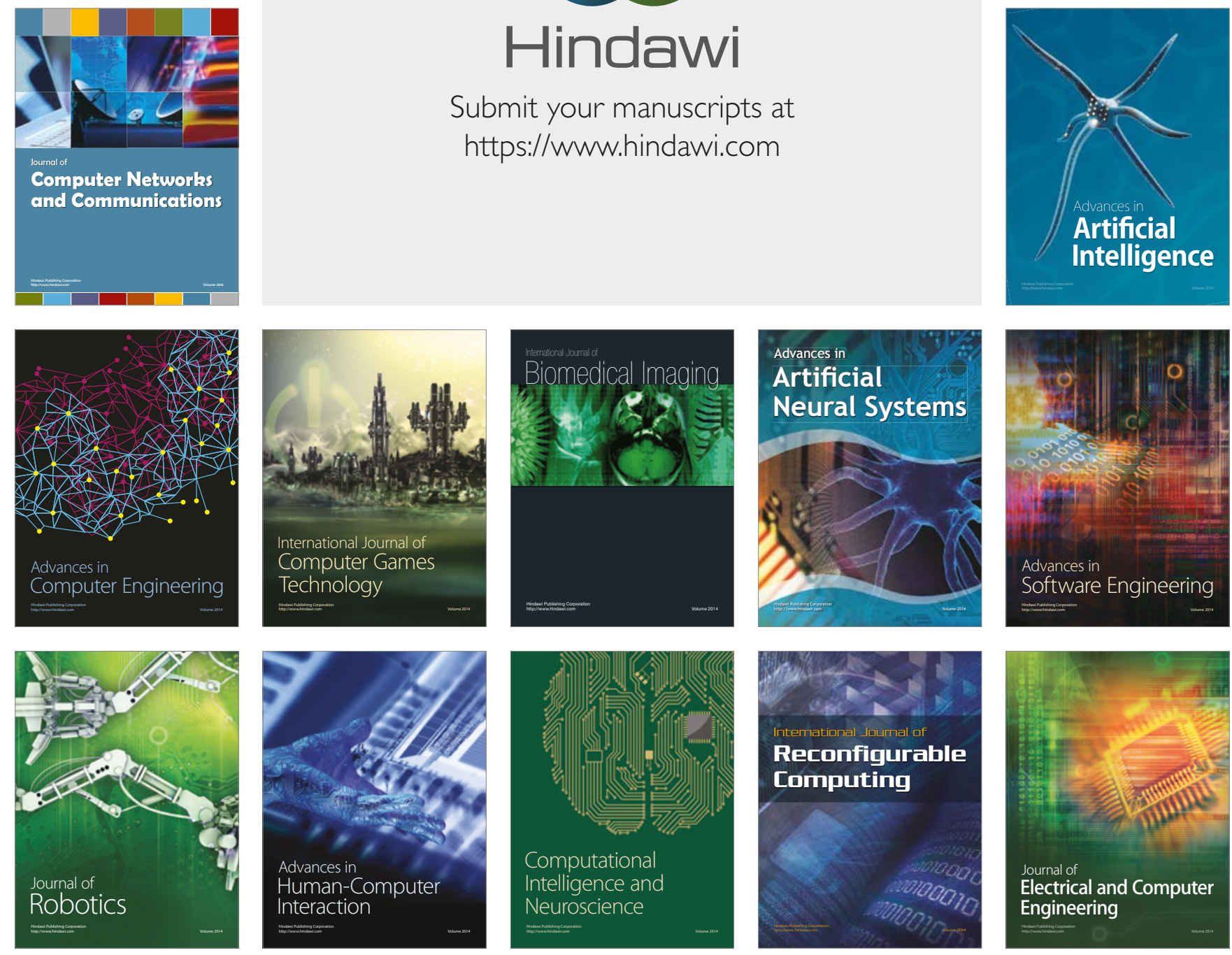